\title{
1 Brucella effector hijacks endoplasmic reticulum quality control machinery to prevent
}

\section{2 premature egress}

3

4 Jean-Baptiste Luizet $^{1}$, Julie Raymond ${ }^{1}$, Thais Lourdes Santos Lacerda ${ }^{1}$, Magali Bonici ${ }^{1}$,

5 Frédérique Lembo $^{2}$, Kévin Willemart ${ }^{3}$, Jean-Paul Borg ${ }^{2}$, Jean-Pierre Gorvel ${ }^{4}$, Suzana P.

6 Salcedo \#1 $^{\# 1}$

7

8

$9{ }^{1}$ Laboratory of Molecular Microbiology and Structural Biochemistry, Centre National de la 10 Recherche Scientifique UMR5086, Université de Lyon, Lyon, France.

11

$12{ }^{2}$ CRCM, Inserm, Institut Paoli-Calmettes, Aix-Marseille Université, CNRS, Marseille,

13 France

14

$15{ }^{3}$ Research Unit in Microorganisms Biology, University of Namur, B-5000 Namur, Belgium

16

$17{ }^{4}$ Aix-Marseille Univ, CNRS, INSERM, CIML, Marseille, France

$22{ }^{\#}$ Corresponding author and lead contact: $\underline{\text { suzana.salcedo@ibcp.fr }}$ 


\section{Abstract}

26

27 Perturbation of endoplasmic reticulum (ER) functions can have critical consequences for

28 cellular homeostasis. An elaborate surveillance system known as ER quality control (ERQC)

29 ensures that only correctly assembled proteins reach their destination. Persistence of

30 misfolded or improperly matured proteins upregulates the unfolded protein response (UPR) to

31 cope with stress, activates ER associated degradation (ERAD) for delivery to proteasomes for

32 degradation. We have identified a Brucella abortus type IV secretion system effector called

33 BspL that targets Herp, a key component of ERQC and is able to augment ERAD.

34 Modulation of ERQC by BspL results in tight control of the kinetics of autophagic Brucella-

35 containing vacuole formation, preventing premature bacterial egress from infected cells. This

36 study highlights how bacterial pathogens may hijack ERAD components for fine regulation of

37 their intracellular trafficking.

38

39 Keywords: Brucella, ERAD, trafficking, Herp, ERQC 


\section{Introduction}

44 The endoplasmic reticulum (ER) is the largest organelle in the cell and plays numerous

45 functions vital for maintaining cellular homeostasis. It is the major site for protein synthesis

46 of both secreted and integral membrane proteins as well as exporting of newly synthesised

47 proteins to other cellular organelles. Disturbance or saturation of the folding-capacity of the

48 ER leads to a complex stress response that has evolved to help cells recover homeostasis or, if

49 necessary, commit them to death. The ER relies on a complex surveillance system known as

50 ER quality control (ERQC) that ensures handling of misfolded, misassembled or

51 metabolically regulated proteins (Braakman and Bulleid, 2011). Once retained in the ER,

52 these proteins are retrotranslocated back into the cytosol to be ubiquitinated and degraded by

53 the proteasome, a process known as ER-associated degradation (ERAD) (Wu and Rapoport,

54 2018). Alternatively, ERAD-resistant proteins can be degraded via ERQC-autophagy (Houck

55 et al., 2014).

57 In response to ER perturbations, particularly following the accumulation of toxic amounts of 58 misfolded proteins, ER stress ensues and cells activate a set of inter-connected pathways that 59 are collectively referred to as the unfolded protein response (UPR) that have a critical role in 60 restoring homeostasis (Walter and Ron, 2011). The UPR is regulated by three ER membrane 61 sensors, the inositol-requiring enzyme I (IRE1), double-stranded RNA-activated protein 62 kinase-like ER kinase (PERK) and activating transcription factor 6 (ATF6). In non-stress 63 conditions these are kept inactive thanks to their association with the ER chaperone BiP. 64 Upon stress, BiP is dislodged from the luminal domains of the three sensors which leads to 65 their activation and induction of specialized transcriptional programs. The IRE1 and ATF6 66 pathways are involved in induction of the transcription of genes encoding for protein-folding 67 chaperones and ERAD-associated proteins (Hetz and Papa, 2018). Whereas PERK sensing is 
68 particularly important in control of autophagy, protein secretion and apoptosis (Hetz and

69 Papa, 2018).

70

71 The homocysteine-inducible ER stress protein (Herp) is an ER membrane protein that is

72 highly upregulated during ER stress by all UPR branches (Kokame et al., 2000; Ma and

73 Hendershot, 2004). Herp is a key component of ERQC that plays a protective role in ER

74 stress conditions (Chan et al., 2004; Tuvia et al., 2007). It is an integral part of the ERAD

75 pathway, enhancing the protein loading and folding capacities of the ER. In addition, it acts as

76 a hub for membrane association of ERAD machinery components, stabilizing their

77 interactions with substrates at ERQC sites (Leitman et al., 2014) and facilitating their

78 retrotranslocation (Huang et al., 2014). Furthermore, as Herp is also in a complex with the

79 proteasome it may aid delivery of specific retrotranslocated substrates to the proteasome for

80 degradation (Kny et al., 2011; Okuda-Shimizu and Hendershot, 2007).

82 Given its importance for cellular homeostasis, the ERQC represents a prime target for microbial pathogens. Indeed, a growing number of bacterial pathogens have been shown to 84 hijack ERQC pathways, especially by modulating UPR (Celli and Tsolis, 2014). For example, 85 Legionella pneumophila secretes several effector proteins that repress CHOP, BiP and XBP1s 86 at the translational level, resulting in UPR inhibition and decrease in inflammation 87 (Hempstead and Isberg, 2015). Another pathogen for which modulation of UPR plays a 88 critical role during infection is Brucella spp., a facultative intracellular pathogen that causes brucellosis, a zoonosis still prevalent worldwide. Brucella abortus has been shown to induce

90 UPR (de Jong et al., 2012; Smith et al., 2013), and more specifically the IRE1 pathway, 91 contributing to enhanced inflammation, a process particularly relevant in the context of 92 colonization of the placenta and abortion (Keestra-Gounder et al., 2016). However, activation 
93 of IRE1 is also important for Brucella trafficking and subsequent Brucella multiplication (Qin

94 et al., 2008; Smith et al., 2013). After cellular uptake, Brucella is found in a membrane bound

95 compartment designated endosomal Brucella-containing vacuole (eBCV) which transiently

96 interacts with early and late endosomes, undergoing limited fusion with lysosomes (Starr et

97 al., 2008). Bacterial are then able to sustain interactions with ER exit sites (ERES) a process

98 that requires the activity of the small GTPases Sar1 (Celli et al., 2005) and Rab2 (Fugier et

99 al., 2009) and results in the establishment of an ER-derived compartment suited for

100 multiplication (replicative or rBCV). UPR induction by Brucella is necessary for this

101 trafficking step, as the formation of rBVCs is dependent on IRE1 activation by the ERES-

102 localized protein Yip1A, which mediates IRE1 phosphorylation and dimerization (Taguchi et

103 al., 2015). Once rBCVs are established, Brucella is capable of extensive intracellular

104 replication, without induction of cell death. Instead, at late stages of the intracellular cycle,

$105 \mathrm{rBCVs}$ reorganize and fuse to form large autophagic vacuoles (aBCVs) that will mediate

106 bacterial exit from infected cells (Starr et al., 2011). The bacterial factors behind the switch

107 between $\mathrm{rBCVs}$ and aBCVs remain uncharacterized.

108

109 Brucella relies on a type 4 secretion system (T4SS), encoded by the virB operon and induced

110 during eBCV trafficking to translocate bacterial effectors into host cells and directly modulate

111 cellular functions. However, only a few effectors have been characterized and for which we

112 have a full grasp of how they contribute towards pathogenesis. This system has been

113 implicated in the induction of UPR during infection and a subset of these effectors has been

114 shown to modulate ER-associated functions. VceC interacts with the ER chaperone BiP to

115 activate the IRE1 pathway, which results in NOD1/NOD2 activation and up-regulation of

116 inflammatory responses (de Jong et al., 2012; Keestra-Gounder et al., 2016). BspA, BspB and

117 BspF have all been implicated in blocking of ER secretion (Myeni et al., 2013). In particular, 
118 BspB was shown to interact with the conserved oligomeric Golgi (COG) complex to redirect

119 vesicular trafficking towards the rBCVs (Miller et al., 2017). Several other effectors that

120 localize in the ER when ectopically expressed have been shown to induce UPR or control ER

121 secretion, but the mechanisms involved remain uncharacterized.

123 In this study, we identify a new T4SS effector of Brucella abortus, that we designate as

124 Brucella-secreted protein L (BspL) that targets a component of the ERAD machinery, Herp.

125 BspL enhances ERAD and delays the formation of aBCVs, preventing early bacterial release

126 from infected cells which helps maintain cell to cell spread efficiency.

\section{Results}

\section{BspL is a Brucella T4SS effector protein}

132 Bacterial effectors are often similar to eukaryotic proteins or contain domains and motifs that

133 are characteristic of eukaryotic proteins. Multiple bacterial effectors benefit from the host

134 lipidation machinery for targeting eukaryotic membranes. Some of these contain a carboxyl-

135 terminal CAAX tetrapeptide motif ( $\mathrm{C}$ corresponds to cysteine, A to aliphatic amino acids and

$136 \mathrm{X}$ to any amino acid) that serves as a site for multiple post-translation modifications and

137 addition of a lipid group which facilitates membrane attachment, such as SifA from

138 Salmonella enterica (Boucrot et al., 2003; Reinicke, 2005) and AnkB from Legionella

139 pneumophila (Price et al., 2010). Previous work highlighted several Brucella encoded

140 proteins that contain putative CAAX motifs (Price et al., 2010) which could therefore be

141 T4SS effectors. In this study, we focused on one of these proteins encoded by the gene

142 BAB1_1533 (YP_414899.1), that we have designated BspL for Brucella-secreted protein L. 
144 We first determined if BspL was translocated into host cells during infection. We constructed 145 a strain expressing BspL fused to the C-terminus of the TEM1 ß-lactamase (encoded by bla) 146 and infected RAW macrophage-like cells for different time-points. A Flag tag was also

147 included for control of protein expression. The fluorescent substrate CCF2 was added and the 148 presence of fluorescent emission of coumarin, resulting from cleavage by the cytosolic TEM1 149 lactamase, was detected by confocal microscopy. This assay is widely used in the Brucella 150 field and we included the T4SS effector VceC as a positive control (de Jong et al., 2008), 151 which showed the highest level of secretion at $24 \mathrm{~h}$ post-infection in our experimental 152 conditions (Figure 1A). We found that TEM1-BspL was secreted into host cells as early as $4 \mathrm{~h}$ 153 post-infection, with a slight peak at $12 \mathrm{~h}$ post-infection, CCF2 cleavage was still detected at $15424 \mathrm{~h}$ post-infection (Figure 1A). This phenotype was fully dependent on the T4SS as a $\triangle v \operatorname{ir} B 9$ 155 mutant strain did not show any coumarin fluorescence (Figure 1A and B). This was not due to 156 lack of expression of TEM1-BspL as both the wild-type and the $\Delta v i r B 9$ strains carrying the 157 bla::bspL plasmid showed equivalent levels of TEM1-BspL expression (Figure 1C). 158 Together, these results show BspL is a T4SS effector.

160 Ectopically expressed BspL accumulates in the ER, does not interfere with host protein 161 secretion but induces the UPR

162 BspL is very well conserved in the Brucella genus, it is 170 amino acids long (Figure S1A) 163 and is approximately $19 \mathrm{kDa}$. BspL does not share any homology to eukaryotic proteins nor 164 to other bacterial effectors. Its nucleotide sequence encodes for a sec secretion signal, a 165 feature commonly found in other Brucella effectors (Marchesini et al., 2011). In addition, it 166 contains a hydrophobic region that may constitute a transmembrane domain as well as a 167 proline rich region, with seven consecutive prolines that may be relevant in interactions with 
168 eukaryotic proteins. To gain insight into the function of BspL we ectopically expressed HA,

169 myc or GFP-tagged BspL in HeLa cells. We found BspL accumulated in the ER, as can be

170 seen by the co-localization with calnexin (Figure $2 \mathrm{~A}$ and $\mathrm{S} 1 \mathrm{~B}, \mathrm{~S} 1 \mathrm{C}$ ), an ER membrane

171 protein and chaperone. Unlike what has been reported for VceC (de Jong et al., 2012), the

172 structure of the ER remained relatively intact upon BspL expression. Deletion of the C-

173 terminal tetrapeptide sequence, which could correspond to a potential lipidation motif had no

174 effect on the ER localization of BspL in transfection (Figure S1B, bottom panel), as it

175 significantly overlapped with the full-length protein when co-expressed in the same cell

176 (Figure S1C).

177

178 Our observations suggest BspL is part of a growing number of Brucella effectors that

179 accumulate in the ER when ectopically expressed, including VceC, BspB and BspD (de Jong

180 et al., 2012; Myeni et al., 2013). We therefore investigated if BspL shared any of the ER

181 modulatory functions described for other effectors, notably interference with ER secretion as

182 BspB (Miller et al., 2017; Myeni et al., 2013) or induction of ER stress as VceC (de Jong et

183 al., 2012; Keestra-Gounder et al., 2016).

184 To determine the impact of BspL on host protein secretion we used the secreted embryonic

185 alkaline phosphatase (SEAP) as a reporter system. HEK cells were co-transfected with the

186 vector encoding SEAP and vectors encoding different Brucella effectors. We chose to work

187 with HA-BspL, to allow direct comparison with previously published HA-BspB that blocks

188 ER secretion and HA-BspD as a negative control (Myeni et al., 2013). Expression of the

189 GDP-locked allele of the small GTPase Arf1[T31N], known to block the early secretory

190 pathway, was used as a control for efficient inhibition of secretion (Figure S1D). As

191 previously reported, we found that expression of HA-BspB drastically reduced SEAP

192 secretion (Figure S1D). In contrast, HA-BspL did not impact SEAP secretion to the same 
193 extent as BspB, having an effect equivalent to HA-BspD previously reported not to affect host

194 protein secretion (Myeni et al., 2013).

195

196 We next investigated whether ER targeting of BspL was accompanied with activation of the

197 UPR, an important feature of Brucella pathogenesis. In the case of B. abortus, IRE1 is the

198 main pathway activated (de Jong et al., 2012) which leads to splicing of the mRNA encoding

199 the transcription factor X-box-binding protein 1 (XBP1) which in turn induces the expression

200 of many ER chaperones and protein-folding enzymes. The second branch of the UPR

201 dependent on PERK may also be of relevance in Brucella infection (Smith et al., 2013).

202 Under prolonged stress conditions, this UPR branch leads to the up-regulation of the

203 transcription factor C/EBP-homologous protein (CHOP) which induces expression of genes

204 involved apoptosis. We therefore monitored XBP1s and CHOP transcript levels following

205 ectopic expression of HA-BspL, in comparison to HA-VceC, established as an ER stress

206 inducer and HA-BspB, known not to induce ER stress. Treatment with tunicamycin, a

207 chemical ER stress inducer was also included. We found that over-expression of HA-BspL

208 induced an increase of both $X B P 1 s$ and $C H O P$ transcription, to levels even higher than HA-

209 VceC (Figure 2B and C). These results suggest BspL may induce ER stress.

210

211 BspL is not involved in establishment of an ER-derived replication niche but is

\section{2 implicated in induction of ER stress during infection}

213 As UPR has been implicated in the establishment of rBCVs (Taguchi et al., 2015) and

214 intracellular replication (Qin et al., 2008; Smith et al., 2013; Taguchi et al., 2015) of Brucella

215 we next investigated the intracellular fate of a $B$. abortus 2308 strain deleted for $b s p L$ in

216 comparison with the wild-type. Two cellular models were used, HeLa cells and an

217 immortalized cell line of bone marrow-derived macrophages (iBMDM). We found that the 
$218 \Delta b s p L$ strain replicated as efficiently as the wild-type in both iBMDM (Figure S2A) and

219 HeLa cells (Figure S2B). In terms of intracellular trafficking no obvious differences were

220 observed in the establishment of rBCVs at 24 and $48 \mathrm{~h}$ post-infection, as $\triangle b s p L \mathrm{BCV}$ s were

221 nicely decorated with the ER marker calnexin in both cell types (Figure 2D and E) as

222 observed for the wild-type strain (Figure S2C and D). As this is the first report to our

223 knowledge to use iBMDM in Brucella infections, we confirmed this observation by

224 quantifying the percentage of BCVs positive for calnexin and the lysosomal associated

225 membrane protein 1 (LAMP1) in comparison with the wild-type at 24 and 48 post-infection

226 (Figure S2E and F, respectively). The wild-type strain in this cellular model behaved as 227 expected forming the typical rBCVs.

228

229 As in transfected cells we found that BspL induced UPR, we next monitored the levels of $230 X B P 1 s$ and $C H O P$ transcripts during infection. Since the rate of infected cells is too low to 231 detect ER stress in HeLa cells, these experiments were only performed in iBMDMs. As 232 expected, the wild-type $B$. abortus strain induced an increase in the levels of transcription of $233 X B P 1 s$ in relation to the mock-infected control iBMDM at $48 \mathrm{~h}$ post-infection (Figure $2 \mathrm{~F}$ ). In 234 contrast, $\triangle b s p L$ infected macrophages showed decreased XBP1s transcript levels compared to 235 the wild-type (Figure 2G). Furthermore, the wild-type phenotype could be fully restored by 236 expressing a chromosomal copy of $b s p L$ in the $\triangle b s p L$ strain, confirming that BspL 237 specifically contributes towards induction of the IRE1 branch of the UPR during infection 238 (Figure 2F). We did not observe an increase in $C H O P$ transcript levels in iBMDM infected 239 with the wild-type nor $\Delta b s p L$ strains in comparison to the mock-infected cells (Figure 2G), 240 suggesting that $B$. abortus does not significantly induce the PERK-dependent branch of the 241 UPR at this stage of the infection. 


\section{BspL interacts with Herp, a key component of ERQC}

244 To gain insight into the function of BspL we set out to identify its interacting partners. A 245 yeast two-hybrid screen identified 7 candidates: eukaryotic translation initiation factor 4A2

246 (EIF4A2), pyruvate dehydrogenase beta (PDHB), MTR 5-methyltetrahydrofolate-

247 homocysteine methyltransferase, Bcl2-associated athanogene 6 (BAG6), ARMCX3 armadillo

248 repeat containing protein (Alex3), homocysteine-inducible ER protein with ubiquitin like

249 domain (Herpud or Herp) and Ubiquilin2 (Ubqln2).

251 In view of our previous results for BspL showing ER localization and induction of UPR we decided to focus on Alex3, Herp and Ubiquilin2 which are rarely present or even absent in the database of false positives for this type of screen (http://crapome.org/). Alex3 is a mitochondrial outer membrane protein that has been implicated in regulation of mitochondrial trafficking (Serrat et al., 2013). As ER and mitochondria extensively interact, Alex3 could constitute an interesting target. Herp is an ER membrane protein playing a role in both the

257 UPR and the ERAD system whereas Ubiquilin2 is implicated in both the proteasome and ERAD and, interestingly, shown to interact with Herp (Kim et al., 2008). In view of these different targets we decided to carry out an endogenous co-immunoprecipitation in cells expressing HA-BspL. As controls for detecting non-specific binding, we also performed co-

261 immunoprecipitations from cells expressing two other ER-targeting effectors, HA-BspB and

262 HA-VceC. We then probed the eluted samples with antibodies against Alex3, Ubiquilin2 or

263 Herp to detect if any interactions could be observed. We found that Alex3 was co264 immunoprecipitated with all 3 effectors suggesting a potentially non-specific interaction with 265 the effectors or the resin itself (Figure 3A). In contrast, no interactions were observed with

266 Ubiquilin2, which was detected only in the flow through fractions. However, we found that 267 endogenous Herp specifically co-immunoprecipated with HA-BspL and not the other 
268 effectors (Figure 3A), suggesting Herp and BspL form a complex within host cells. Taken

269 together with the yeast two-hybrid data, we can conclude that BspL directly interacts with

270 Herp. Consistently, over-expressed BspL co-localized with Herp by microscopy (Figure 3B).

271

272 BspL facilitates degradation of TCR $\alpha$ via ERAD independently of ER stress

273 Herp is a key component of ERAD, strongly up-regulated upon ER stress. Indeed, during $B$.

274 abortus infection we observed an up-regulation of HERP transcripts (Figure S3A), consistent

275 with $X B P 1 s$ induction, although these differences were not statistically significant with the

276 number of replicates carried out. However, inhibition of Herp using siRNA (Figure S3B)

277 showed that ER stress induced following ectopic expression of BspL was not dependent on

278 Herp (Figure S3C and D), suggesting BspL interaction with Herp is mediating other functions

279 in the cell.

280

281 Therefore, we next investigated if BspL could directly impact ERAD. We used expression of

$282 \mathrm{~T}$ cell receptor alpha $(\mathrm{TCR} \alpha)$ as reporter system, as this type I transmembrane glycoprotein

283 has been shown to be a canonical ERAD substrate, quickly degraded (Feige and Hendershot,

284 2013; Lippincott-Schwartz et al., 1988). TCR $\alpha$ is transferred across the ER membrane, where

285 is becomes glycosylated and fails to assemble. This in turn induces its retrotranslocation back

286 to the cytosol to be degraded by the proteasome. Cycloheximide treatment for $4 \mathrm{~h}$ was used to

287 block protein synthesis, preventing replenishment of TCR pools and allowing for

288 visualization of ERAD-mediated degradation of TCR $\alpha$. When HEK-293T cells, which do not

289 naturally express TCR were transfected with HA-TCR $\alpha$ and treated with cycloheximide, a

290 decrease in HA-TCR $\alpha$ was observed, indicative of degradation (Figure 4A, red arrow).

291 Strikingly, expression of BspL induced very strong degradation of TCR $\alpha$ (Figure 4A). This is

292 accompanied by the appearance of a faster migrating band at around $25 \mathrm{KDa}$ (blue arrow), 
293 that nearly disappears upon cycloheximide treatment suggesting this TCR $\alpha$ peptide is

294 efficiently degraded by the proteasome. It is important to note that the $25 \mathrm{KDa}$ band is also

295 present when HA-TCR $\alpha$ is expressed alone (lane 2 of Figure 4A, blue arrow) suggesting it is

296 a natural intermediate of HA-TCR $\alpha$ degradation.

297

298 To determine if the enhanced effect of BspL on TCR $\alpha$ degradation is a side-effect of ER

299 stress, cells were treated with TUDCA which strongly inhibited both XBP1s and CHOP

300 transcript levels induced by either tunicamycin, BspL or VceC (Figure S3E and F). In the

301 presence of TUDCA, BspL was still found to enhance HA-TCR $\alpha$ degradation showing this is

302 occurring in an ER stress-independent manner (Figure S4).

303

304 As the TCR $\alpha$ subunit undergoes N-glycosylation in the ER, we wondered if the faster

305 migrating band of TCR $\alpha$ induced by BspL corresponded to non-glycosylated form of TCR $\alpha$.

306 We therefore treated samples with EndoH, which deglycosylates peptides. Upon EndoH

307 treatment we observed deglycosylated HA-TCR $\alpha$ (second lane, Figure 4B, black arrow),

308 confirming the reporter system is being processed normally. In the BspL expressing samples

309 (lanes 3 and 4, Figure 4B), a slight band corresponding to the non-glycosylated TCR $\alpha$ could

310 also be detected particularly after EndoH treatment, confirming that BspL does not prevent

311 TCR $\alpha$ from entering the ER and being glycosylated. The dominant TCR $\alpha$ band induced upon

312 BspL expression (around $25 \mathrm{KDa}$, blue arrow) migrates faster than the non-glycosylated form

313 resulting from EndoH treatment (black arrow) and does not appear to be sensitive to EndoH.

314 This may therefore correspond to a natural truncated non-glycosylated form of HA-TCR $\alpha$.

315 Consistently, this band is also present in the absence of BspL (lane 1, Figure 4B, blue arrow).

316 Together these data indicate that BspL is a strong inducer of ERAD. 


\section{ERAD is required for different stages of intracellular lifecycle of Brucella}

319 The role of ERAD in the Brucella intracellular life cycle has not yet been investigated to our

320 knowledge. We therefore decided to block ERAD using eeyarestatin, an established inhibitor

321 of this system. Unfortunately, prolonged treatment at the concentration necessary for full

322 inhibition of ERAD induced detachment of infected iBMDM. Nonetheless, we were able to

323 carry out this experiment in HeLa cells, which showed significant resistance to the

324 eeyarestatin treatment. Total CFU counts after addition of eeyarestatin at $2 \mathrm{~h}$ post-infection

325 showed a significant decrease in bacterial counts at $48 \mathrm{~h}$, suggesting a potential inhibition of

326 replication (Figure 5A). However, microscopy observation of infected cells at this time-point

327 clearly showed extensive replication of bacteria even in the presence of eeyarestatin (Figure

328 5B), suggesting that the drop of CFU observed was a result of exit of bacteria from infected

329 cells rather than inhibition of intracellular replication. Consistently, we observed significant

330 numbers of extracellular bacteria as well as many cells infected with only a few bacteria

331 potentially resulting from re-infection. These results suggest that blocking of ERAD during

332 early stages of infection would favour intracellular replication. To confirm this possibility, we

333 counted by microscopy the number of bacteria per cell at $24 \mathrm{~h}$ post-infection and indeed found

334 a higher replication rate upon eeyarestatin treatment (Figure 5C). We therefore hypothesized

335 that Brucella might block ERAD during early stages of the infection to favour establishment

336 of an early replication niche, a phenotype clearly not dependent on BspL, as we have shown it

337 is not implicated in the establishment of rBCVs and when ectopically expressed it induces

338 ERAD. We therefore, wondered if BspL could intervene at a later stage of the infection to 339 induce ERAD via its interaction with Herp.

340

341 BspL delays premature bacterial egress from infected cells 
342 The late stage of the intracellular cycle of Brucella relies on induction of specific autophagy

343 proteins to enable the formation of aBCVs characterized as large vacuoles with multiple

344 bacteria decorated with LAMP1 (Starr et al., 2011). In our experimental conditions aBCVs

345 could be clearly observed in iBMDM infected for $65 \mathrm{~h}$ with wild-type B. abortus (Figure 6A).

346 We therefore investigated if BspL was involved in formation of aBCVs. Strikingly, $\triangle b s p L$

347 aBCVs could be detected as early as $24 \mathrm{~h}$, with nearly $30 \%$ of infected cells showing aBCVs

348 at $48 \mathrm{~h}$ post-infection compared to less than $10 \%$ for wild-type infected cells (Figure $6 \mathrm{~B}$ and

349 C). Importantly, complementation of the $\Delta b s p L$ strain fully restored the wild-type phenotype.

350 These results strongly suggest that BspL is involved in delaying the formation of aBCVs

351 during $B$. abortus macrophage infection. Consistently, imaging of $\triangle b s p L$ infected iBMDM at

$35248 \mathrm{~h}$, revealed the presence of high numbers of extracellular bacteria as well as cells with

353 single bacteria or a single aBCV (Figure 6D), suggestive of re-infection and reminiscent of

354 what was observed following eeyarestatin treatment that blocks the ERAD. In contrast, wild-

355 type infected iBMDM at the same time-point showed none or few signs of re-infection with

356 most cells showing extensive perinuclear ER-like distribution of bacteria (Figure 6D).

357 In conclusion, we propose that, secretion of BspL during Brucella infection induces ERAD to

358 control aBCV formation and prevent premature bacterial egress from infected cells.

361 Discussion

362

363 In this study, we characterize a previously unknown T4SS effector of B. abortus and its role

364 in virulence. We found this effector hijacks the ERAD machinery to regulate the late stages of

365 the Brucella intracellular cycle. Although many bacterial pathogens have been shown to

366 control UPR, very little is known about the impact of ERAD, a downstream process following 
367 UPR, in the context of intracellular bacterial infections. To our knowledge there are only two

368 examples. The obligatory intracellular pathogen Orientia tsutsugamushi, the cause of scrub

369 thypus, is an auxotroph for histidine and aromatic amino acids and was shown to transiently

370 induce UPR and block ERAD during the first 48h of infection (Rodino et al., 2017). This in

371 turn enables release of amino acids in the cytosol, necessary for its growth (Rodino et al.,

372 2017). The second example is Legionella pneumophila, that recruits the AAA ATPase

373 Cdc48/p97 to its vacuole, that normally recognizes ubiquitinated substrates and can act as a

374 chaperone in the context of ERAD to deliver misfolded proteins to the proteasome.

375 Recruitment of Cdc48/p97 to the Legionella vacuole is necessary for intracellular replication

376 and helps dislocate ubiquitinated proteins from the vacuolar membrane, including bacterial

377 effectors (Dorer et al., 2006).

378

379 In the case of BspL we found it directly interacts with Herp, a component of ERAD which is

380 induced upon UPR. Our data suggest that BspL enhances ERAD and this prompted us to

381 further investigate the role of ERAD during Brucella infection. Interestingly, we found that

382 inhibition of ERAD is beneficial during early stages of intracellular trafficking and enhances

383 bacterial multiplication. It is possible that Brucella is transiently blocking ERAD during

384 rBCV formation and initial replication, potentially via a specific set of effectors or a particular

385 cellular signal yet to be identified. This could, as demonstrated for Orientia, release amino

386 acids into the cytosol that would be critical for bacterial growth. Alternatively, or in parallel, a

387 block of ERAD could potentially enhance autophagy to deal with the ER stress that would in 388 turn favour rBCV formation.

390 As a permanent block of ERAD could become damaging to the cell under prolonged stress

391 and, as we observed, speed up the bacterial release from infected cells potentially 
392 prematurely, Brucella translocation of BspL could counteract these effects by enhancing

393 ERAD and slowing down aBCV formation. We could not directly show BspL ERAD

394 induction is dependent on Herp as its depletion would itself block ERAD (Hori et al., 2004;

395 Okuda-Shimizu and Hendershot, 2007). However, in the presence of BspL no glycosylated

396 ER loaded HA-TCR $\alpha$ was observed indicative of enhanced processing through the ERAD

397 pathway. Instead, only a truncated unglycosylated TCR $\alpha$ intermediate was detected, which

398 disappeared in the presence of cycloheximide suggesting it is efficiently degraded. These

399 likely correspond to a backlog of peptides awaiting proteasomal degradation, generated by an

400 abnormal ERAD flux induced by BspL.

401

402 Further work is now required to establish the precise mechanisms that enables BspL to

403 facilitate ERAD. It is possible that BspL interaction with Herp stabilizes it, preventing its

404 degradation and would therefore help sustain ERAD. Indeed, ER stress significantly induces

405 Herp levels but Herp was shown to be quickly degraded, enabling efficient modulation of

406 ERQC (Yan et al., 2014). Alternatively, BspL may favour Herp accumulation at ERQC sites

407 that would also enhance its ability to assist protein retrotranslocation and delivery to

408 proteasomes. Imaging of BspL during infection will help to determine if a particular sub-ER

409 compartment is targeted, such as ERQC-sites.

410

411 This study focuses on BspL-Herp interactions, nevertheless we cannot exclude the

412 participation of other potential targets identified in the yeast-two hybrid screen, notably

413 Ubiquilin 2 and Bag6. Ubiquilins function as adaptor proteins between the proteasome and

414 ubiquination machinery and therefore participate in ERAD. Ubiquilins also interact with Herp

415 (Kim et al., 2008) and very interestingly have been shown to play a role in control of 416 autophagy (Şentürk et al., 2019). Our co-immunoprecipitation experiment did not reveal any 
417 binding but perhaps a weak or transient interaction is taking place not detectable with our

418 current in vitro conditions. Another interesting target is Bag6, (also known as Bat3) a

419 chaperone of the Hsp70 family that is also involved in delivery of proteins to the ER or when

420 they are not properly folded to the proteasome. Bag6 was shown to be the target of the

421 Orientia Ank4 effector that blocks ERAD (Rodino et al., 2017) and to be targeted by multiple

422 Legionella effectors to control host cell ubiquitination processes (Ensminger and Isberg, 423 2010). Therefore, it is possible that Bag6 may contribute towards BspL control of ERAD 424 functions during Brucella infection.

425

426 In addition to ERAD, we found that BspL itself was implicated in induction of UPR.

427 However, this phenotype was independent of Herp and may be an indirect effect due to its ER 428 accumulation or via another cellular target yet to be characterized. Furthermore, the increased 429 ERAD activity upon BspL expression was not a result of increased ER stress; suggesting that 430 BspL is independently controlling these two pathways. There is growing evidence that the 431 induction of IRE1-dependent UPR by multiple effectors is linked to modulation of Brucella 432 intracellular trafficking and intracellular multiplication (Smith et al., 2013; Taguchi et al., 433 2015). Our data allow us to add another piece to this complex puzzle, and place for the first 434 time the ERAD pathway at the centre of Brucella regulation of its intracellular trafficking. 435 Further work is now required to decipher all the molecular players involved.

437 In conclusion, our results show that ERAD modulation by BspL enables Brucella to 438 temporarily delay the formation of aBCVs and avoid premature egress from infected cells, 439 highlighting a new mechanism for fine-tuning of bacterial pathogen intracellular trafficking. 


\section{Acknowledgements}

443 This work was funded by the ERA-Net Pathogenomics CELLPATH grant (ANR 2010-PATH444 006), the FINOVI foundation under a Young Researcher Starting Grant and the ANR charm$445 E d$ (grant $\mathrm{n}^{\circ}$ ANR-18-CE15-0003), both obtained by SPS. JBL was supported by a doctoral 446 contract from the Région Rhônes-Alpes ARC1 Santé. SPS is supported by an INSERM staff 447 scientist contract. We are very grateful to Linda Hendershot (St Judes Medical School, USA) 448 for sending us the pcDNA-TCR $\alpha$ and for all the help with setting up the ERAD assay and 449 discussion of the results. We thank Renée Tsolis (University of California at Davis, USA) and 450 Jean Celli (Washington State University, USA) with advice for the construction of the 451 following plasmids TEM1-VceC, HA-VceC, HA-BspB and HA-BspD, as the French Agency 452 ANSM has prevented us from importing these vectors directly from them due to the size of 453 the genes encoded. We also thank Thomas Henry (CIRI, Lyon, France) for the iBMDM. A 454 final special thanks to Jean Celli (Washington State University, USA) for sending us several 455 protocols and vectors (pSEAP and pmini-Tn7 vectors) as well as providing us constant 456 guidance for the SEAP assay, complementation and observation of aBCVs. The two-hybrid 457 screening was hosted by the Marseille Proteomics platform (JPB, FL) supported by Institut 458 Paoli-Calmettes, IBISA (Infrastructures Biologie Santé et Agronomie), Aix-Marseille 459 University, Canceropôle PACA and the Région Sud Provence-Alpes-Côte d'Azur. JPB is a 460 scholar of Institut Universitaire de France. We thank Steve Garvis, Amandine Blanco and 461 Arthur Louche for critical reading of the manuscript.

\section{Author contributions}

464 Conceptualization: JBL, JPB, JPG and SPS. Investigation: JBL, JR, TLSL, MB, FL, KW and

465 SPS; Writing of Original Draft: JBL and SPS; Writing, Review \& Editing: all authors; 466 Funding Acquisition: SPS. 


\section{Declaration of Interests}

469 The authors declare no competing interests.

470

\section{$471 \quad$ Figure Legends}

472 Figure 1. BspL is a T4SS effector translocated into host cells during B. abortus infection.

473 (A) Macrophage-like cell line (RAW) was infected with B. abortus carrying a plasmid 474 encoding for bla fused with BspL (pbla::bspL) to enable expression of TEM-BspL. Cells 475 were infected with either wild-type B. abortus or $\triangle$ virB9 carrying this plasmid. A positive 476 control of wild-type expressing bla::vceC was included. At 4, 12 or 24 h post-infection, cells

477 were incubated with fluorescent substrate CCF2-AM, fixed and the percentage of cells with 478 coumarin emission quantified using an automated plugin. More than a 1000 cells were 479 quantified for each condition from 3 independent experiments and data represent means \pm 480 standard deviations. Kruskal-Wallis with Dunn's multiple comparisons test was used and $\mathrm{P}=$ 4810.0019 between wild-type pbla::bspL and $\Delta v i r B 9$ pbla::bspL at $12 \mathrm{~h}\left({ }^{* *}\right)$ and 0.171 at $24 \mathrm{~h}(*)$. 482 Not all statistical comparisons are shown.

483 (B) Representative images of cells infected for 24 h with B. abortus wild-type or $\Delta v i r B 9$ 484 carrying pbla::bspL. Cells were incubated with CCF2 and the presence of translocated 485 TEM1-BspL detected by fluorescence emission of coumarin (red). Scale bars correspond to 5 $486 \mu \mathrm{m}$.

487 (C) The expression of TEM1-BspL in the inocula of wild-type and $\triangle v i r B 9$ strains was 488 controlled by western blotting thanks to the presence of a FLAG tag in the construct. The membrane was probed with an anti-Flag antibody (top) or anti-Omp25 (bottom) as a loading

490 control. A sample from wild-type without the plasmid was included as a negative control. 491 Molecular weights are indicated (KDa). 
493 Figure 2. BspL does not impact early BCV trafficking but contributes to UPR induction 494 at late stages of the infection.

495 (A) Confocal microscopy image showing the intracellular localization of HA-BspL expressed 496 in HeLa cells labelled with an anti-HA antibody (green) and ER marker calnexin (red). 497 Phalloidin (cyan) was used to label the actin cytoskeleton and Dapi (white) for the nucleus.

498 (B) Quantification of mRNA levels of XBP1s and (C) CHOP by quantitative RT-PCR 499 obtained from HeLa cells expressing HA-BspL, HA-VceC or HA-BspB for 24h. Cells 500 transfected with empty vector pcDNA3.1 were included as a negative control and cells treated 501 tunicamycin at $1 \mu \mathrm{g} / \mu \mathrm{l}$ for $6 \mathrm{~h}$ as a positive control. Data correspond to the fold increase in 502 relation to an internal control with non-transfected cells. Data are presented as means \pm 503 standard deviations from at least 4 independent experiments. Kruskal-Wallis with Dunn's 504 multiple comparisons test was used and $\mathrm{P}=0.042$ between negative and HA-BspL (**) and 5050.0383 between HA-BspL and HA-BspB (*) for XBP1s. For $C H O P, \mathrm{P}=0.0184$ between 506 negative and tunicamycin (*); 0.0088 between negative and HA-BspL (**); 0.0297 bteween 507 tunicamycin and HA-BspB (*) and 0.011 between HA-BspL and HA-BspB (*). All other 508 comparisons ranked non-significant.

509 (D) Representative images of rBCVs from $\triangle b s p L$-expressing DSred infected iBMDM or (E) 510 HeLa cells at 24 and 48h post-infection, labelled for calnexin (green).

511 (F) Quantification of mRNA levels of XBP1s and (G) CHOP by quantitative RT-PCR 512 obtained from iBMDMs infected with wild-type, $\triangle b s p L$ or the complemented $\Delta b s p L:: b s p L$ 513 strains for $48 \mathrm{~h}$. Mock-infected cells were included as a negative control. Data correspond to 514 the fold increase in relation to an internal control with non-infected cells. Data are presented 515 as means \pm standard deviations from at least 3 independent experiments. Kruskal-Wallis with 516 Dunn's multiple comparisons test was used and, for XBP1s, $\mathrm{P}=0.042$ between negative and 
517 HA-BspL (**) and 0.0352 between the negative control and wild-type infected cells (*) and

5180.0111 between negative and the complemented $\triangle b s p L:: b s p L$ infected cells $(*)$. All other

519 comparisons ranked non-significant with this test.

520

521 Figure 3. BspL specifically interacts with the ERAD component Herp.

522 (A) Co-immunoprecipitation (co-IP) from cell extracts expressing either HA-BspL, HA-BspB

523 and HA-VceC using HA-trapping beads. Flow through and elutions were probed with

524 antibodies against Alex3, Ubiquilin (Ubqln) and Herp in succession. The level of each

525 effector bound to the beads was revealed with an anti-HA antibody and $15 \%$ of the input used

526 for the co-IP shown (at the bottom). Molecular weights are indicated (KDa).

527 (B) Representative confocal micrograph of HeLa cells expressing HA-BspL (green) and 528 labelled for Herp (red). Scale bar corresponds to $5 \mu \mathrm{m}$.

$530 \quad$ Figure 4. BspL enhances ERAD degradation of TCR $\alpha$.

531 (A) HEK $293 \mathrm{~T}$ cells were transfected with HA- TCR $\alpha$ in the absence or presence of myc-

$532 \mathrm{BspL}$ for $24 \mathrm{~h}$. Where indicated, cells were treated with $50 \mu \mathrm{g} / \mathrm{ml}$ cycloheximide for the last

533 4h. The blot was probed first with an anti-TCR antibody followed by anti-actin. The same

534 samples were loaded onto a separate gel (separated by dashed line) for probing with an anti-

535 myc and anti-actin to confirm the expression of myc-BspL. Molecular weights are indicated

$536(\mathrm{KDa})$ and relevant bands described in the text highlighted with different coloured arrows.

537 (B) HEK $293 \mathrm{~T}$ cells were transfected with HA- TCR $\alpha$ in the absence or presence of myc-

538 BspL for 24h and samples treated with EndoH where indicated. The blot was probed first

539 with an anti-TCR antibody followed by anti-actin. The same samples were loaded onto a

540 separate gel (separated by dashed line) for probing with an anti-myc and anti-actin to confirm 
541 the expression of myc-BspL. Molecular weights are indicated (KDa) and relevant bands

542 described in the text highlighted with different coloured arrows.

543

544 Figure 5. Blocking of ERAD at early stages of the infection enhances intracellular 545 replication and accelerates bacterial release.

546 (A) Bacterial counts (CFU) at 2, 24 and 48h post-infection with either the wild-type without 547 any treatment (wt, black) or in the presence of $8 \mu \mathrm{M}$ eeyarestatin (wt+Eeya, red) or the 548 equivalent amount of DMSO (wt+DMSO, green). Data correspond to means \pm standard 549 deviations from 6 independent experiments. A two-way ANOVA was used yielding a $\mathrm{P}<$ $5500.0001(* * * *)$ between wild-type+DMSO with wild-type+Eeya at $48 \mathrm{~h}$. Other comparisons are 551 not indicated.

552 (B) Representative confocal images of HeLa cells infected with the wild-type DSRed or 553 following treatment eeyarestatin at $48 \mathrm{~h}$ post-infection.

554 (C) Microscopy bacterial counts at $24 \mathrm{~h}$ post-infection with either the wild-type with DMSO 555 or in the presence of $8 \mu \mathrm{M}$ eeyarestatin. Data is presented as the percentage of cells 556 containing 1 to 5 bacteria per cell (red), 6 to 30 (black), 30 to 40 (blue) or more than 50

557 (green). Data correspond to means \pm standard deviations from 3 independent experiments. A 558 two-way ANOVA test was used yielding a $\mathrm{P}=0.0003(* * *)$ between wild-type+DMSO with 559 wild-type+Eeya at $48 \mathrm{~h}$. Other comparisons are not indicated.

560

561 Figure 6. BspL is implicated in delay of aBCV formation.

562 (A) Representative confocal images of iBMDM infected with wild-type DSred for $65 \mathrm{~h}$ 563 labelled for LAMP1 (green). Scale bar corresponds to $5 \mu \mathrm{m}$.

564 (B) Representative confocal images of iBMDM infected with $\triangle b s p L$ DSred for $24 \mathrm{~h}$ (top), $48 \mathrm{~h}$ 565 (middle) and 65h (lower), labelled for LAMP1 (green). Scale bars correspond to $5 \mu \mathrm{m}$. 
566 (C) Quantification of the percentage of cells with aBCVs, in iBMDMs infected with either

567 wild-type, $\triangle b s p L$ or the complemented $\triangle b s p L:: b s p L$ strains for 24,48 or $65 \mathrm{~h}$. Data

568 correspond to means \pm standard deviations from at least 5 independent experiments. A two-

569 way ANOVA was used yielding a $\mathrm{P}<0.0001(* * * *)$ between wild-type and $\triangle b s p L$ as well as

$570 \Delta b s p L$ and $\triangle b s p L:: b s p L$ at $48 \mathrm{~h}$. Other comparisons are not indicated.

571 (D) Representative confocal image of iBMDM infected with either wild-type DSRed or $572 \Delta b s p L$ for $48 \mathrm{~h}$, labelled for calnexin (red). Bacteria shown in white. Scale bars correspond to $5735 \mu \mathrm{m}$.

574

575

Supplementary Figure Legends

576

577 Figure S1. BspL targets the ER independently of its CAAX motif without impacting ER 578 secretion.

579 (A) Schematic diagram of BspL and its domains, namely the Sec secretion signal, 580 hydrophobic region, Prolin-rich region (PRR) and potential CAAX motif with amino acid C, $581 \quad \mathrm{~T}, \mathrm{~A}$ and $\mathrm{N}$.

582 (B) Representative confocal images of HeLa cells expressing myc-BspL (top panel) or myc583 BspL $\triangle$ CAAX (bottom panel) labelled for the ER marker calnexin (red). Scale bars 584 correspond to $5 \mu \mathrm{m}$.

585 (C) HeLa cells were co-transfected with GFP-BspL (green) and myc-BspL $\triangle$ CAAX (cyan) for $58624 \mathrm{~h}$ and labelled for the ER marker calnexin (red). Scale bars correspond to $5 \mu \mathrm{m}$.

587 (D) Quantification of SEAP secretion in HEK 293T cells expressing either control empty 588 vector (pcDNA3.1), dominant negative form of Arf1 (HA-ARF[T31N]), HA-BspL, HA, 589 BspB or HA-BspD. Measurements were done at $24 \mathrm{~h}$ after transfection and the secretion index 590 corresponds to means \pm standard deviations. Kruskal-Wallis with Dunn's multiple 
comparisons test was used and $\mathrm{P}=0.0164$ between pcDNA control and HA-ARF[T31N] $(*)$

592 and 0.0005 between pcDNA and HA-BspB (***). All other comparisons ranked non-

593 significant.

594

595 Figure S2. Equivalent intracellular trafficking of wild-type and $b s p L$ mutant strains.

596 (A) Bacterial counts using colony forming units (CFU) at 2, 24 and 48h post-infection with

597 either the wild-type (red) or $\triangle b s p L$ strains (black) of iBMDM or (B) HeLa cells. Data

598 correspond to means \pm standard deviations from 3 independent experiments.

599 (C) iBMDM or (D) HeLa cells were infected with wild-type B. abortus DSRed (red) for 24 or

$60048 \mathrm{~h}$ and labelled for the ER marker calnexin (green). Zoomed insets are indicated. Scale bars

601 correspond to $5 \mu \mathrm{m}$.

602 (E) Quantification of the percentage of BCVs positive for calnexin or (F) LAMP1 at 24 or $60348 \mathrm{~h}$ post-infection of iBMDM with either wild-type or $\Delta b s p L$ DSRed-expressing strains. Data 604 are presented as means \pm standard deviations from at 6 independent experiments. Kruskal-

605 Wallis with Dunn's multiple comparisons test was used and all comparisons between the 606 wild-type and the mutant strain yielded $\mathrm{P}>0.05$, considered as non-significant.

607

608 Figure S3. BspL induction of ER stress is independent of Herp.

609 (A) Quantification of mRNA levels of HERP by quantitative RT-PCR obtained from 610 iBMDMs infected with wild-type, $\triangle b s p L$ or the complemented $\Delta b s p L:: b s p L$ strains for $48 \mathrm{~h}$.

611 Mock-infected cells were included as a negative control. Data correspond to the fold increase

612 in relation to an internal control with non-infected cells. Data are presented as means \pm 613 standard deviations from 3 independent experiments. Kruskal-Wallis with Dunn's multiple 614 comparisons test was used and yielded non-significant differences. 
615 (B) Western blot of cell lysates from HeLa cells treated with siRNA control (siCtrl) or siRNA

616 Herp (siHerp) for 48h. A sample from non-treated cells was included as a negative control.

617 Membrane was probed with an anti-Herp antibody followed by anti-actin for loading control.

618 (C) Quantification of mRNA levels of XBP1s or (D) CHOP by quantitative RT-PCR obtained

619 from HeLa cells expressing HA-BspL or HA-VceC for $24 \mathrm{~h}$. Where indicated, HeLa cells

620 were treated with siRNA control (siCtrl) or siRNA Herp (siHerp). Cells transfected with

621 empty vector pcDNA3.1 were included as a negative control and cells treated tunicamycin at

$6221 \mu \mathrm{g} / \mu \mathrm{l}$ for $6 \mathrm{~h}$ as a positive control. Data correspond to the fold increase in relation to an

623 internal control with non-transfected cells. Data are presented as means \pm standard deviations

624 from at least 3 independent experiments. Kruskal-Wallis with Dunn's multiple comparisons

625 test was used and yielded $\mathrm{P}=0.0184(*)$ between negative siCtrl and BspL siCtrl, $0.0277(*)$

626 between negative siHerp and BspL siHerp and $0.0485(*)$ between negative siCtrl and

627 tunicamycin siCtrl. No significant differences for observed for $C H O P$.

628 (E) Quantification of mRNA levels of XBP1s or (F) CHOP by quantitative RT-PCR obtained 629 from HeLa cells expressing HA-BspL, HA-VceC or HA-BspB for 24h. Were indicated, cells 630 were treated with $0.5 \mathrm{nM}$ of TUDCA for $22 \mathrm{~h}$. Cells transfected with empty vector pcDNA3.1

631 were included as a negative control and cells treated tunicamycin at $1 \mu \mathrm{g} / \mu \mathrm{l}$ for $6 \mathrm{~h}$ as a 632 positive control. Data correspond to the fold increase in relation to an internal control with 633 non-transfected cells. Data are presented as means \pm standard deviations from 3 independent 634 experiments. Kruskal-Wallis with Dunn's multiple comparisons test was used and yielded $635 \mathrm{P}=0.0439(*)$ between $\mathrm{BspL}$ and BspL+TUDCA. For CHOP, $\mathrm{P}=0.0012$ (**) between 636 tunicamycin and tunicamycin+TUDCA, $0.0036(* *)$ between BspL and BspL+TUDCA and $6370.0192(*)$ between $\mathrm{VceC}$ and VceC+TUDCA. Not all comparisons are indicated. 
640 HEK 293T cells were transfected with HA- TCR $\alpha$ in the absence or presence of myc-BspL

641 for $24 \mathrm{~h}$. Where indicated, cells were treated with $50 \mu \mathrm{g} / \mathrm{ml}$ cycloheximide for the last $6 \mathrm{~h}$ or

$6420.5 \mathrm{nM}$ of TUDCA for $22 \mathrm{~h}$. The blot was probed first with an anti-TCR antibody followed by

643 anti-actin. The same samples were loaded onto a separate (separated by dashed line) for

644 probing with an anti-myc and anti-actin to confirm the expression of myc-BspL. Molecular

645 weights are indicated (KDa) and relevant bands described in the text highlighted with 646 different coloured arrows.

647

648

\section{Material and methods}

650

\section{Cell culture}

652 HeLa, RAW and HEK293T cells obtained from ATCC were grown in DMEM supplemented with $10 \%$ of fetal calf serum. Immortalized bone marrow-derived macrophages from

654 C57BL/6J mice were obtained from Thomas Henry (CIRI, Lyon, France) and were maintained in DMEM supplemented with 10\% FCS and 10\% spent medium from L929 cells

656 that supplies MC-CSF.

657

\section{Transfections and siRNA}

659 All cells were transiently transfected using Torpedo® (Ibidi-Invitrogen) for $24 \mathrm{~h}$, according to 660 manufacturer's instructions. siRNA experiments were done with Lipofectamine® RNAiMAX

661 Reagent (Invitrogen) according the protocol of the manufacturers. Importantly, siRNA 662 depletion of Herp was done by treatment with $3 \mu \mathrm{M}$ siRNA the day after seeding of cells and 663 again at $24 \mathrm{~h}$. Depletion was achieved after $48 \mathrm{~h}$ total. Depletion was confirmed by western 664 blotting with an antibody against Herp. ON-TARGETplus siRNA SMARTpool (L-020918) 
665 were used for Herp and for the control ON-TARGETplus Non-targeting pool (D-001810)

666 both from from Dharmacon. For both transfections and siRNA cells were weeded 18h before

667 at $2 \times 10^{4}$ cells/well and $1 \times 10^{5}$ cells/well for 24 and 6 well plates, respectively.

668

669 Bacterial strains and growth conditions

670 Brucella abortus 2308 was used in this study. Wild-type and derived strains were routinely

671 cultured in liquid tryptic soy broth and agar. $50 \mu \mathrm{g} / \mathrm{ml}$ kanamycin was added for cultures of

672 DSRed or complemented strains.

673

674 Construction of BspL eukaryotic expression vectors

675 The BspL constructs were obtained by cloning in the gateway pDONR ${ }^{\mathrm{TM}}$ (Life Technologies)

676 and then cloned in the pENTRY Myc, HA or GFP vectors. The following primers were used

677 5'-GGGGACAAGTtTGTACAAAAAAGCAGGCTTCAATCGATtTTtGAAGATCACTAT-3' and 5'-

678 GGGGACCACTTTGTACAAGAAAGCTGGGTCCTAGTTGGCCGTGCAGAAATG-3'. For the construct

679 without CAAX the following reverse primer was used: 5'680 GGGGACCACTTtGTACAAGAAAGCTGGGTCCTAGAAATGGTCGCGACCGTCA-3'. The final 681 constructs were verified by sequencing and expression of tagged-BspL verified by western 682 blotting.

683

\section{Construction of $b s p L$ mutant and complementing strain}

685 B. abortus 2308 knockout mutant $\triangle b s p L$ was generated by allelic replacement. Briefly, 686 upstream and downstream regions of about $750 \mathrm{bp}$ flanking the $b s p L$ gene were amplified by 687 PCR (Q5 NEB) from B. abortus 2308 genomic DNA using the following primers: (i) 688 SpeI_Upstream_Forward: actagtATGTCGAGAACTGCCTGC,

689 BamHI_XbaI_Upstream_Reverse:

CGGGATCCCGGCTC 
690 TAGAGCGCGGCTCCGATTAAAACAG,

(iii) BamHI_XbaI_Downstream_Forward:

691 CGGGATCC CGGCTCTAGAGCACCGAACCGATCAACCAG and

692 SpeI_Downstream_Reverse: actagtCC CTATACCGAGTTGGAGC. A joining PCR was used

693 to associate the two PCR products using the following primers pairs: (i) and (iv). Finally, the

$694 \Delta B s p L$ fragment was cloned in a SpeI digested suicide vector (pNPTS138). The acquisition of

695 this vector by $B$. abortus after mating with conjugative S17 Escherichia coli was selected

696 using the kanamycin resistance cassette of the pNPTS138 vector and the resistance of $B$.

697 abortus to nalidixic acid. The loss of the plasmid concomitant with either deletion of a return

698 to the wild type phenotype was then selected on sucrose, using the $s a c B$ counter selection

699 marker also present on the vector. Deletant $(\Delta)$ strain was identified by diagnostic PCR using

700 the following primers: Forward: CACTGGCAATGATCAGTTCC and Reverse:

701 CTGACCATTATGTGTGAACAGG (Amplicon length: WT-2000 bp, $\Delta$ - 1500 bp).

702 The complementing strain was constructed by amplifying BspL and its promoter region (500

703 bp upstream) with the PrimeStar DNA polymerase (Takara) using the following primers: Fw:

704 AAAGGATCCGACAATCAGAAGGTTTCCTATGAAACG and Rev:

705 AAAACTAGTTCAGTTGGCCGTGCAGAAATG. Insert and pmini-Tn7 (Myeni et al.,

706 2013) were digested with BamHI and SpeI and ligated overnight. Transformants were

707 selected on kanamycin $50 \mu \mathrm{g} / \mathrm{mL}$ and verified by PCR and sequencing. To obtain the

708 complementing strain the $\Delta b s p L$ mutant was electroporated with pmini-Tn7-bspL with the

709 helper plasmid pTNS2. Electroporants were selected on tryptic soy agar plates with

710 kanamycin $50 \mu \mathrm{g} / \mathrm{mL}$ and verified by PCR.

711

712 HA-TCR $\alpha$

713 The pcDNA-TCR $\alpha$ was obtained from Linda Hendershort (St Judes Medical School, USA)

714 and it corresponds to the A6-TCR $\alpha$ (Feige and Hendershot, 2013). The HA tag was 
715 introduced by sequence and ligation independent cloning (SLIC) method with the following

716 primers:

TCR-Fw:

717

718

CGAGCTCGGATCCACTAGTCCAGTGTGGTGGAATTCTACCCATACGATGTTCCAG

and

TCR-

Rv:GAGCGGCCGCCACTGTGCTGGATATCTGCAGAATTCTTACTAGCTAGACCACA

721

G. Briefly, pcDNA-TCR $\alpha$ was digested with EcoRI and incubated with purified PCR product

amplified with the PrimeStar DNA polymerase (Takara - Ozyme) for 3 min at RT followed

by $10 \mathrm{~min}$ on ice. The following ratio was used for the reaction: $100 \mathrm{ng}$ vector $+3 \mathrm{x}$ PCR insert.

\section{Infections}

Bacterial cultures were incubated for $16 \mathrm{~h}$ from isolated colonies in TSB shaking overnight at

$37{ }^{\circ} \mathrm{C}$. Culture optical density was controlled at $600 \mathrm{~nm}$. Bacterial cultures diluted to obtain the appropriate multiplicity of infection (MOI) for HeLa 1:500 and iBMDMs 1:300 in the appropriate medium. Infected cells were centrifuged at $400 \mathrm{x}$ g for 10 minutes to initiate bacterial-cell contact followed by incubation for $1 \mathrm{~h}$ at $37^{\circ} \mathrm{C}$ and $5 \% \mathrm{CO}_{2}$ for $\mathrm{HeLa}$ cells and only 15 min for iBMDMs. After the cells were washed 3 times with DMEM and treated with

732 gentamycin $(50 \mu \mathrm{g} / \mathrm{mL})$ to kill extracellular bacteria for $1 \mathrm{~h}$. At 2 hours pi the medium was 733 replaced with a weaker gentamycin concentration $10 \mu \mathrm{g} / \mathrm{mL}$. Cells are plated $18 \mathrm{~h}$ before 734 infection and seeded at $2 \times 10^{4}$ cell / well and $1 \times 10^{5}$ cells/well for 24 and 6 well plates respectively. For qRT-PCR experiments, $10 \mathrm{~mm}$ cell culture plates were used at a density of $1 \times 10^{6}$ cell/plate. At the different time points cells were either harvested of coverslips fixed for

737 immunostaining. In the case of bacterial cell counts, cells were lysed in $0.1 \%$ Triton for 5 min 738 and a serial dilution plated for enumeration of bacterial colony forming units (CFU). 


\section{Immunofluorescence microscopy}

741 At the appropriate time point, coverslips were washed twice with PBS, fixed with AntigenFix

742 (MicromMicrotech France) for 15 minutes and then washed again 4 times with PBS. For ER

743 and Herp immunostaining, permeabilization was carried out with a solution of PBS

744 containing $0.5 \%$ saponin for 30 minutes followed by blocking also for 30 minutes in a

745 solution of PBS containing $1 \%$ bovine serum albumin (BSA), $10 \%$ horse serum, $0.5 \%$

746 saponin, $0.1 \%$ Tween and $0.3 \mathrm{M}$ glycine. Coverslips were then incubated for $3 \mathrm{~h}$ at room

747 temperature or at $4{ }^{\circ} \mathrm{C}$ overnight with primary antibody diluted in the blocking solution.

748 Subsequently, the coverslips were washed twice in PBS containing $0.05 \%$ saponin and

749 incubated for $2 \mathrm{~h}$ with secondary antibodies. Finally, coverslips were washed twice in PBS

750 with $0.05 \%$ saponin, once in PBS and once in ultrapure water. Lastly, they were mounted on

751 a slide with ProLongGold (Life Technologies). The coverslips were visualized with a

752 Confocal Zeiss inverted laser-scanning microscope LSM800 and analyzed using ImageJ

753 software. For Lamp1 immunostaining no pre-permeabilization and blocking were done and

754 coverslips were directly incubated with antibody mix diluted in PBS containing 10\% horse

755 serum and $0.5 \%$ saponin for $3 \mathrm{~h}$ at room temperature. The remaining of the protocol was the

756 same as described above.

757

$758 \quad$ Western blotting

759 Cells were washed 1x with PBS and the 1x with ice-cold PBS. Cells were scrapped ince-cold

760 PBS, centrifuged for $5 \mathrm{~min}$ at $4{ }^{\circ} \mathrm{C}$ at $80 \mathrm{~g}$. Pellets where then ressuspended in cell lysis buffer

761 (Chromotek) supplemented with phenylmethylsulfonyl fluoride (PMSF) and proteinase

762 inhibitors tablet cocktail (complete Mini, Roche). Samples resolved on SDS-PAGE and

763 transferred onto PVDF membrane Immobilon-P (Millipore) using a standard liquid transfer

764 protocol. Membranes were blocked using PBS with $0.1 \%$ Tween 20 and 5\% skim milk for 30 
765 min and the probed using relevant primary antibodies overnight at $4{ }^{\circ} \mathrm{C}$, washed 3 times with

766 PBS with $0.1 \%$ Tween 20 and then incubated with HRP-conjugated secondary anti-goat,

767 mouse or rabbit antibodies, diluted in PBS with Tween $200.1 \%$ and 5\% skim milk for $1 \mathrm{~h}$.

768 Western blots were revealed using ECL Clarity reagent (BioRad). Signals were acquired

769 using a Fusion Camera and assembled for presentation using Image J.

770

771 TEM1 translocation assay

772 RAW cells were seeded in a 96 well plates at $1 \times 10^{4}$ cells/well overnight. Cells were then

773 infected with an MOI of 300 by centrifugation at $4{ }^{\circ} \mathrm{C}, 400 \mathrm{~g}$ for $5 \mathrm{~min}$ and 1 at $37{ }^{\circ} \mathrm{C} 5 \%$

$774 \mathrm{CO}_{2}$. Cells were washed with HBSS containing $2.5 \mathrm{mM}$ probenicid. Then $6 \mu 1$ of CCF2 mix

775 (as described in the Life Technologies protocol) and $2.5 \mathrm{mM}$ probenicid were added to each

776 well, and incubated for $1.5 \mathrm{~h}$ at room temperature in the dark. Cells were finally washed with

777 PBS, fixed using Antigenfix and analysed immediately by confocal microscopy (Zeiss

778 LSM800).

779

780

\section{RNA isolation and real-time quantitative polymerase chain reaction (qRT-PCR)}

781 HeLa cells were seeded in $100 \times 100$ culture dishes at $1 \times 10^{6}$ cells/plate for each condition and

782 were either transfected with HA-tagged BspL, VceC or BspB for $24 \mathrm{~h}$ or infected with wild-

783 type, mutant or complemented strains for $48 \mathrm{~h}$. Cells were then washed $1 \mathrm{x}$ in PBS, scrapped in

784 buffer RLT (Qiagen) supplemented with ß-mercaptoethanol and transfered on a Qiashredder

785 column (Qiagen). Then several wash steps were performed and total RNAs were extracted

786 using a RNeasy Mini Kit (Qiagen). 500 ng of RNA were reverse transcribed in a final volume

787 of $20 \mu \mathrm{l}$ using QuantiTect Reverse Transcription Kit (Qiagen). Real-time PCR was performed

788 using SYBR Green PowerUp (ThermoScientific) with an QuantiTect Studio 3

789 (ThermoScientific). Specific primers for human cells: HERP fw: 
790 CGTTGTTATGTACCTGCATC and HERP rev: TCAGGAGGAGGACCATCATTT ; XBP $1 s$

791 fw: TGCTGAGTCCGCAGCAGGTG and XBP1s rev: GCTGGCAGGCTCTGGGGAAG;

792 CHOP fw: GCACCTCCCAGAGCCCTCACTCTCC and CHOP rev:

793 GTCTACTCCAAGCCTTCCCCCTGCG. The HPRT, and GAPDH expressions were used as

794 internal controls for normalization and fold change calculated in relation to the negative

795 control. Primers were HPRT fw: TATGGCGACCCGCAGCCCT and HPRT rev:

796 CATCTCGAGCAAGACGTTCAG; GAPDH fw: GCCCTCAACGACCACTTTGT and

797 GAPDH rev: TGGTGGTCCAGGGGTCTTAC.

798 For murine cells: HERP fw: CAACAGCAGCTTCCCAGAAT and HERP rev:

799 CCGCAGTTG GAGTGTGAGT; XBP1s fw: GAGTCCGCAGCAGGTG and XBP1s rev:

800 GTGTCAGAGTCCATGGGA; $C H O P$ fw: CTGCCTTTCACCTTGGAGAC and $C H O P$ rev:

801 CGTTTCCTGGGGATGAGATA and for the internal controls for normalization primers were

$80218 S$ fw: GTAACCCGTTGAACCCCATT and $18 S$ rev: CCATCCAATCGGTAGTAGCG;

803 GAPDH fw: TCACCACCATGGAGAAGGC and GAPDH rev:

804 GCTAAGCAGTTGGTGGTGCA. Data were analyzed using Prism Graph Pad 6.

805

806 ERAD evaluation

807 HEK293T cells seeded in $100 \mathrm{~mm}$ culture plates at $8 \times 10^{5}$ cells/plate overnight and then co-

808 transfected for $24 \mathrm{~h}$ with Torpedo (Ibidi) with vectors encoding HA-TCR $(5 \mu \mathrm{g})$ and myc-

809 BspL $(5 \mu \mathrm{g})$. Cycloheximide $50 \mu \mathrm{g} / \mathrm{ml}$ was added $6 \mathrm{~h}$ before lysis. Where indicated, TUDCA

810 was added $2 \mathrm{~h}$ after transfection at $0.5 \mathrm{mM}$. Cells were harvested as described above (western

811 blotting) and lysed in $200 \mu \mathrm{l}$ of lysis buffer (Chromotek). EndoH (New England Biolabs)

812 treatment was carried out following the manufacturers protocol for $1 \mathrm{~h}$ at $37^{\circ} \mathrm{C}$. Sample buffer

813 was then added (30 mM Tris- $\mathrm{HCl} \mathrm{pH} 6.8,1 \%$ SDS, $5 \%$ glycerol, $0.025 \%$ bromophenol blue 
814 and 1.25 ß-mercaptoethanol final concentration). Western blotting was done as described

815 above using anti-TCR antibody. Actin levels were also analyzed as a loading control.

816

\section{Secretion assay}

818 HEK293T cells were harvested and seeded in 6-well plates at $1 \times 10^{5}$ cells/well and co-

819 transfected with plasmids encoding Brucella secreted proteins (300 ng DNA) and the secreted 820 embryonic alkaline phosphatase (SEAP) (300 ng DNA) provided by Jean Celli. Total amount 821 of transfected DNA was maintained constant using an empty vector pcDNA 3.1 for the 822 positive control. At $18 \mathrm{~h}$ post transfection, the transfection media was removed and then cells 823 were still incubated at $37^{\circ} \mathrm{C} 5 \% \mathrm{CO}_{2}$. Fourty-eight hours later, media containing culture 824 supernatant (extracellular SEAP) was removed and collected. To obtain intracellular SEAP, 825 each well was washed with PBS and then incubated with a solution of PBS-Triton X-100 $8260.5 \%$ for 10 minutes. An incubation of each fraction was performed at $65{ }^{\circ} \mathrm{C}$ following a 827 centrifugation at maximum speed for 30 seconds. Then cells were incubated with a provided 828 substrate 3-(4-methoxyspiro [1,2-dioxetane-3,2'(5'-chloro)-tricyclo(3.3.1.13,7) decane]-4829 yl)phenyl phosphate (CSPD) by SEAP reporter gene assay, chemiluminescent kit (Roche 830 Applied Science). Chemiluminescence values were obtained with the use of a TECAN at 492 $831 \mathrm{~nm}$. Data are presented as the SEAP secretion index, which is a ratio of extracellular SEAP 832 activity to intracellular SEAP activity.

\section{Yeast two-hybrid}

835 BspL was cloned into pDBa vector, using the Gateway technology, transformed into MaV203 836 and used as a bait to screen a human embryonic brain cDNA library (Invitrogen). Media, 837 transactivation test, screening assay and gap repair test were performed as described (Orr838 Weaver and Szostak, 1983; Thalappilly et al., 2008; Walhout and Vidal, 2001). 


\section{$840 \quad$ Antibodies}

841 For immunostaining for microscopy the following antibodies were used:

842 Rat anti-HA antibody clone 3F10 (Roche, \#1867423) was used at a dilution 1/50 and mouse 843 anti-HA (Covance, clone 16B12, \#MMS-101R), at 1/500. Rabbit anti-calnexin (Abcam, 844 \#ab22595) was used at 1/250. Rabbit anti-Herp EPR9649 (Abcam, \#ab150424) at 1/250. The 845 mouse anti-myc antibody clone 9E10 (developed by Bishop, J.M.) was used at 1/1000. Rat 846 anti-LAMP1 clone ID4B (developed by August, J.T.) was used 1/100 for mouse cells and 847 mouse anti-LAMP1 clone H4A3 (developed by August, J.T. / Hildreth, J.E.K.) was used 848 1/100 for human cells. All LAMP1 and Myc antibodies were obtained from the

849 Developmental Studies Hybridoma Bank, created by the NICHD of the NIH and maintained 850 at the University of Iowa. Secondary anti-mouse, rabbit and rat antibodies were conjugated 851 with Alexas-555, -488 or -647 fluorochromes all from Jackson Immunoresearch at a dilution 852 1/1000. Phallodin Atto-647 (Sigma, \#65906) was used at a dilution of 1/1000. Dapi nuclear 853 dye (Invitrogen) was used at a dilution of 1/1000.

854 For western blotting the following antibodies were used:

855 rabbit anti-FLAG (Sigma, \#F7425) at 1/1000 ; rabbit anti-Alex3 (Sigma, \# HPA000967) at 856 1/100; rabbit anti-Ubiquilin 2 (Abcam, \#ab217056) at 1/1000; rabbit anti-Herp EPR9649 857 (Abcam, \# ab150424) at 1/1000; mouse anti-HA (Covance, clone 16B12, ref. MMS-101R) at 858 1/1000; rabbit anti-TCR clone 3A8 (Invitrogen, \#TCR1145) at 1/1000; mouse anti-myc 859 antibody clone 9E10 at 1/1000 ; mouse anti-actin AC-40 (Sigma, \#A4700) at 1/1000. Anti860 mouse (GE Healthcare) or rabbit-HRP (Sigma) antibodies were used at 1/5000.

861

\section{Drug treatments}


863 All drug treatments are indicated in the specific protocols. To summarize the concentrations

864 used were: TUDCA (Focus Biomolecules) at $0.5 \mathrm{nM}$; Cycloheximide (Sigma) at $50 \mu \mathrm{g} / \mathrm{ml}$;

865 Eeyarstatin (Sigma) at $8 \mu \mathrm{M}$; Tunicamycin (Sigma) at $1 \mu \mathrm{g} / \mu \mathrm{l}$; Probenicid (Sigma) at 2.5

$866 \mathrm{mM}$.

867

\section{Co-immunoprecipitation}

869 HeLa cells were cultured in $100 \mathrm{~mm}$ x $20 \mathrm{~mm}$ cell culture dishes at $1 \times 10^{6}$ cells/dish 870 overnight. Cells were transiently transfected with $30 \mathrm{uL}$ of Torpedo ${ }^{\text {DNA }}$ (Ibidi) for $24 \mathrm{~h}$ for a

871 total of $10 \mu \mathrm{g}$ of DNA/plate. On ice, after 2 washes with cold PBS cells were collected with a 872 cell scraper and centrifuged at $80 \mathrm{~g}$ at $4{ }^{\circ} \mathrm{C}$ during $10 \mathrm{~min}$. Cell lysis and processing for co-

873 immunoprecipitation were done as described with the PierceTM HA Epitope Antibody 874 Agarose conjugate (Thermo scientific).

875

876 Statistical analysis

877 All data sets were tested for normality using Shapiro-Wilkinson test. When a normal 878 distribution was confirmed a One-Way ANOVA test with a Tukey correction was used for 879 statistical comparison of multiple data sets with one independent variable and a Two-Way 880 ANOVA test for two independent variables. For data sets that did not show normality, a 881 Kruskall-Wallis test was applied, with Dunn's correction, or Mann-Whitney U-test for two 882 sample comparison. All analyses were done using Prism Graph Pad 6.

\section{References}

Boucrot, E., Beuzón, C.R., Holden, D.W., Gorvel, J.-P., Méresse, S., 2003. Salmonella typhimurium SifA effector protein requires its membrane-anchoring C-terminal hexapeptide for its biological function. J Biol Chem 278, 14196-14202. doi:10.1074/jbc.M207901200

Braakman, I., Bulleid, N.J., 2011. Protein folding and modification in the mammalian endoplasmic reticulum. Annu. Rev. Biochem. 80, 71-99. doi:10.1146/annurev-biochem- 
901

902

903

904

905

906

907

908

909

910

911

912

913

914

915

916

917

918

919

920

921

922

923

924

925

926

927

928

929

930

931

932

933

934

935

936

937

938

939

940

062209-093836

Celli, J., Salcedo, S.P., Gorvel, J.-P., 2005. Brucella coopts the small GTPase Sar1 for intracellular replication. Proc Natl Acad Sci USA 102, 1673-1678. doi:10.1073/pnas.0406873102

Celli, J., Tsolis, R.M., 2014. Bacteria, the endoplasmic reticulum and the unfolded protein response: friends or foes? Nature Publishing Group 1-12. doi:10.1038/nrmicro3393

Chan, S.L., Fu, W., Zhang, P., Cheng, A., Lee, J., Kokame, K., Mattson, M.P., 2004. Herp stabilizes neuronal $\mathrm{Ca} 2+$ homeostasis and mitochondrial function during endoplasmic reticulum stress. J Biol Chem 279, 28733-28743. doi:10.1074/jbc.M404272200

de Jong, M.F., Starr, T., Winter, M.G., Hartigh, den, A.B., Child, R., Knodler, L.A., van Dijl, J.M., Celli, J., Tsolis, R.M., 2012. Sensing of bacterial type IV secretion via the Unfolded Protein Response. mBio 4, e00418-12-e00418-12. doi:10.1128/mBio.00418-12

de Jong, M.F., Sun, Y.-H., Hartigh, den, A.B., van Dijl, J.M., Tsolis, R.M., 2008. Identification of VceA and $\mathrm{VceC}$, two members of the $\mathrm{VjbR}$ regulon that are translocated into macrophages by the Brucella type IV secretion system. Mol Microbiol 70, 13781396. doi:10.1111/j.1365-2958.2008.06487.x

Dorer, M.S., Kirton, D., Bader, J.S., Isberg, R.R., 2006. RNA interference analysis of Legionella in Drosophila cells: exploitation of early secretory apparatus dynamics. PLoS Pathog 2, e34-13. doi:10.1371/journal.ppat.0020034

Ensminger, A.W., Isberg, R.R., 2010. E3 Ubiquitin ligase activity and targeting of BAT3 by multiple Legionella pneumophila translocated substrates. Infect Immun 78, 3905-3919. doi:10.1128/IAI.00344-10

Feige, M.J., Hendershot, L.M., 2013. Quality control of integral membrane proteins by assembly-dependent membrane integration. Mol Cell 51, 297-309. doi:10.1016/j.molcel.2013.07.013

Fugier, E., Salcedo, S.P., de Chastellier, C., Pophillat, M., Muller, A., Arce-Gorvel, V., Fourquet, P., Gorvel, J.-P., 2009. The glyceraldehyde-3-phosphate dehydrogenase and the small GTPase Rab 2 are crucial for Brucella replication. PLoS Pathog 5, e1000487. doi:10.1371/journal.ppat.1000487

Hempstead, A.D., Isberg, R.R., 2015. Inhibition of host cell translation elongation by Legionella pneumophila blocks the host cell unfolded protein response. Proc Natl Acad Sci USA 112, E6790-E6797. doi:10.1073/pnas.1508716112

Hetz, C., Papa, F.R., 2018. The Unfolded Protein Response and cell fate control. Molecular Cell 69, 169-181. doi:10.1016/j.molcel.2017.06.017

Hori, O., Ichinoda, F., Yamaguchi, A., Tamatani, T., Taniguchi, M., Koyama, Y., Katayama, T., Tohyama, M., Stern, D.M., Ozawa, K., Kitao, Y., Ogawa, S., 2004. Role of Herp in the endoplasmic reticulum stress response. Genes to Cells 9, 457-469. doi:10.1111/j.1356-9597.2004.00735.x

Houck, S.A., Ren, H.Y., Madden, V.J., Bonner, J.N., Conlin, M.P., Janovick, J.A., Conn, P.M., Cyr, D.M., 2014. Quality control autophagy degrades soluble ERAD-resistant conformers of the misfolded membrane protein GnRHR. Molecular Cell 54, 166-179. doi:10.1016/j.molcel.2014.02.025

Huang, C.-H., Chu, Y.-R., Ye, Y., Chen, X., 2014. Role of HERP and a HERP-related protein in HRD1-dependent protein degradation at the endoplasmic reticulum. Journal of Biological Chemistry 289, 4444-4454. doi:10.1074/jbc.M113.519561

Keestra-Gounder, A.M., Byndloss, M.X., Seyffert, N., Young, B.M., Chávez-Arroyo, A., Tsai, A.Y., Cevallos, S.A., Winter, M.G., Pham, O.H., Tiffany, C.R., de Jong, M.F., Kerrinnes, T., Ravindran, R., Luciw, P.A., McSorley, S.J., Bäumler, A.J., Tsolis, R.M., 2016. NOD1 and NOD2 signalling links ER stress with inflammation. Nature 1-15. doi:10.1038/nature17631 
941 Kim, T.-Y., Kim, E., Yoon, S.K., Yoon, J.-B., 2008. Herp enhances ER-associated protein

942

943

944

945

946

947

948

949

950

951

952

953

954

955

956

957

958

959

960

961

962

963

964

965

966

967

968

969

970

971

972

973

974

975

976

977

978

979

980

981

982

983

984

985

986

987

988

989

990 degradation by recruiting ubiquilins. Biochem Biophys Res Commun 369, 741-746. doi:10.1016/j.bbrc.2008.02.086

Kny, M., Standera, S., Hartmann-Petersen, R., Kloetzel, P.-M., Seeger, M., 2011. Herp regulates Hrd1-mediated ubiquitylation in a ubiquitin-like domain-dependent manner. Journal of Biological Chemistry 286, 5151-5156. doi:10.1074/jbc.M110.134551

Kokame, K., Agarwala, K.L., Kato, H., Miyata, T., 2000. Herp, a new ubiquitin-like membrane protein induced by endoplasmic reticulum stress. J Biol Chem 275, 3284632853. doi:10.1074/jbc.M002063200

Leitman, J., Shenkman, M., Gofman, Y., Shtern, N.O., Ben-Tal, N., Hendershot, L.M., Lederkremer, G.Z., 2014. Herp coordinates compartmentalization and recruitment of HRD1 and misfolded proteins for ERAD. Mol Biol Cell 25, 1050-1060. doi:10.1091/mbc.E13-06-0350

Lippincott-Schwartz, J., Bonifacino, J.S., Yuan, L.C., Klausner, R.D., 1988. Degradation from the endoplasmic reticulum: disposing of newly synthesized proteins. Cell 54, 209220.

Ma, Y., Hendershot, L.M., 2004. Herp is dually regulated by both the endoplasmic reticulum stress-specific branch of the unfolded protein response and a branch that is shared with other cellular stress pathways. J Biol Chem 279, 13792-13799. doi:10.1074/jbc.M313724200

Marchesini, M.I., Herrmann, C.K., Salcedo, S.P., Gorvel, J.-P., Comerci, D.J., 2011. In search of Brucella abortus type IV secretion substrates: screening and identification of four proteins translocated into host cells through VirB system. Cell Microbiol 13, 1261-1274. doi:10.1111/j.1462-5822.2011.01618.x

Miller, C.N., Smith, E.P., Cundiff, J.A., Knodler, L.A., Bailey Blackburn, J., Lupashin, V., Celli, J., 2017. A Brucella type IV effector targets the COG tethering complex to remodel host secretory traffic and promote intracellular replication. Cell Host Microbe 22, 317329.e7. doi:10.1016/j.chom.2017.07.017

Myeni, S., Child, R., Ng, T.W., Kupko, J.J., Wehrly, T.D., Porcella, S.F., Knodler, L.A., Celli, J., 2013. Brucella modulates secretory trafficking via multiple type IV secretion effector proteins. PLoS Pathog 9, e1003556. doi:10.1371/journal.ppat.1003556.s014

Okuda-Shimizu, Y., Hendershot, L.M., 2007. Characterization of an ERAD pathway for nonglycosylated BiP substrates, which require Herp. Molecular Cell 28, 544-554. doi:10.1016/j.molcel.2007.09.012

Orr-Weaver, T.L., Szostak, J.W., 1983. Yeast recombination: The association between double-strand gap

repair and crossing-over. Proc Natl Acad Sci USA 80, 4417-4421.

Price, C.T.D., Al-Quadan, T., Santic, M., Jones, S.C., Abu Kwaik, Y., 2010. Exploitation of conserved eukaryotic host cell farnesylation machinery by an F-box effector of Legionella pneumophila. J Exp Med 207, 1713-1726. doi:10.1084/jem.20100771

Qin, Q.-M., Pei, J., Ancona, V., Shaw, B.D., Ficht, T.A., de Figueiredo, P., 2008. RNAi screen of endoplasmic reticulum-associated host factors reveals a role for IRE1alpha in supporting Brucella replication. PLoS Pathog 4, e1000110. doi:10.1371/journal.ppat.1000110

Reinicke, A.T., 2005. A Salmonella typhimurium effector protein SifA is modified by host cell prenylation and S-acylation machinery. Journal of Biological Chemistry 280, 1462014627. doi:10.1074/jbc.M500076200

Rodino, K.G., VieBrock, L., Evans, S.M., Ge, H., Richards, A.L., Carlyon, J.A., Palmer, G.H., 2017. Orientia tsutsugamushi modulates endoplasmic reticulum-associated degradation to benefit its growth. Infect Immun 86, e00596-17-16. 
doi:10.1128/IAI.00596-17

Serrat, R., López-Doménech, G., Mirra, S., Quevedo, M., Garcia-Fernàndez, J., Ulloa, F., Burgaya, F., Soriano, E., 2013. The non-canonical Wnt/PKC pathway regulates mitochondrial dynamics through degradation of the arm-like domain-containing protein Alex3. PLoS ONE 8, e67773. doi:10.1371/journal.pone.0067773

Smith, J.A., Khan, M., Magnani, D.D., Harms, J.S., Durward, M., Radhakrishnan, G.K., Liu, Y.-P., Splitter, G.A., 2013. Brucella induces an Unfolded Protein Response via TcpB that supports intracellular replication in macrophages. PLoS Pathog 9, e1003785. doi:10.1371/journal.ppat.1003785.s007

Starr, T., Child, R., Wehrly, T.D., Hansen, B., Hwang, S., López-Otin, C., Virgin, H.W., Celli, J., 2011. Selective subversion of autophagy complexes facilitates completion of the Brucella intracellular cycle. Cell Host Microbe 1-14. doi:10.1016/j.chom.2011.12.002

Starr, T., Ng, T.W., Wehrly, T.D., Knodler, L.A., Celli, J., 2008. Brucella intracellular replication requires trafficking through the late endosomal/lysosomal compartment. Traffic 9, 678-694. doi:10.1111/j.1600-0854.2008.00718.x

Şentürk, M., Lin, G., Zuo, Z., Mao, D., Watson, E., Mikos, A.G., Bellen, H.J., 2019. Ubiquilins regulate autophagic flux through mTOR signalling and lysosomal acidification. Nat Cell Biol 1-19. doi:10.1038/s41556-019-0281-x

Taguchi, Y., Imaoka, K., Kataoka, M., Uda, A., Nakatsu, D., Horii-Okazaki, S., Kunishige, R., Kano, F., Murata, M., 2015. Yip1A, a novel host factor for the activation of the IRE1 pathway of the Unfolded Protein Response during Brucella infection. PLoS Pathog 11, e1004747. doi:10.1371/journal.ppat.1004747.s006

Thalappilly, S., Suliman, M., Gayet, O., Soubeyran, P., Hermant, A., Lecine, P., Iovanna, J.L., Dusetti, N.J., 2008. Identification of multi-SH3 domain-containing protein interactome in pancreatic cancer: A yeast two-hybrid approach. Proteomics 8, 30713081. doi:10.1002/pmic.200701157

Tuvia, S., Taglicht, D., Erez, O., Alroy, I., Alchanati, I., Bicoviski, V., Dori-Bachash, M., Ben-Avraham, D., Reiss, Y., 2007. The ubiquitin E3 ligase POSH regulates calcium homeostasis through spatial control of Herp. J Cell Biol 177, 51-61. doi:10.1083/jcb.200611036

Walhout, A.J., Vidal, M., 2001. High-throughput yeast two-hybrid assays for large-scale protein interaction mapping. Methods 24, 297-306. doi:10.1006/meth.2001.1190

Walter, P., Ron, D., 2011. The unfolded protein response: from stress pathway to homeostatic regulation. Science 334, 1081-1086. doi:10.1126/science.1209038

Wu, X., Rapoport, T.A., 2018. Mechanistic insights into ER-associated protein degradation. Curr Opin Cell Biol 53, 22-28. doi:10.1016/j.ceb.2018.04.004

Yan, L., Liu, W., Zhang, H., Liu, C., Shang, Y., Ye, Y., Zhang, X., Li, W., 2014. Ube2g2gp78-mediated HERP polyubiquitylation is involved in ER stress recovery. J Cell Sci 127, 1417-1427. doi:10.1242/jcs.135293 


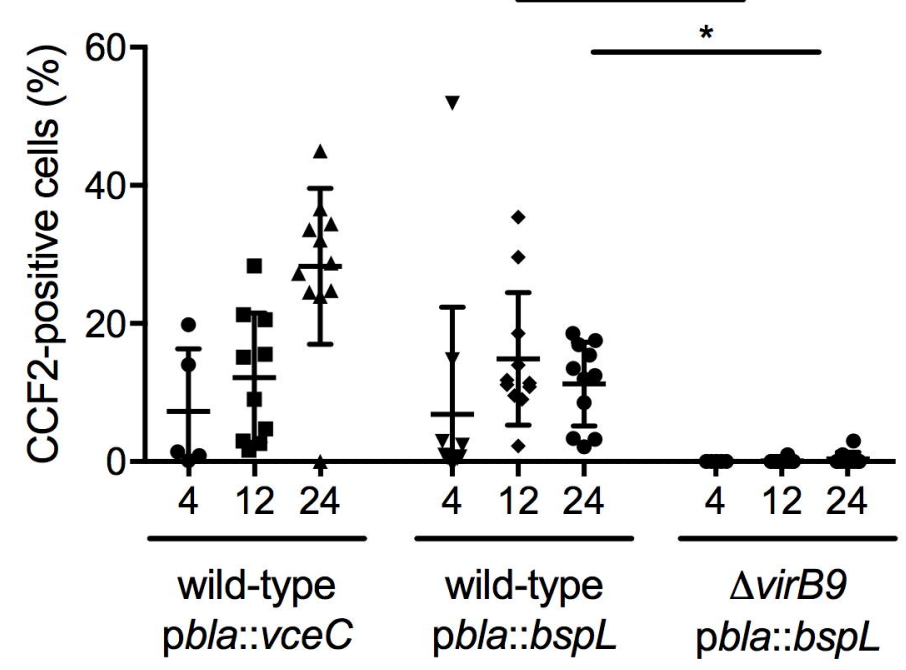

C

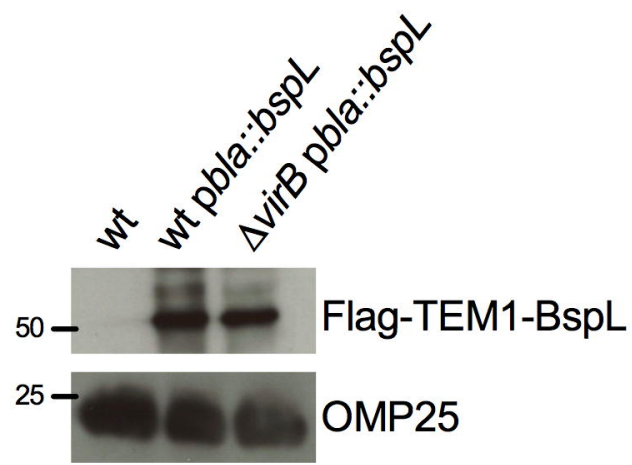

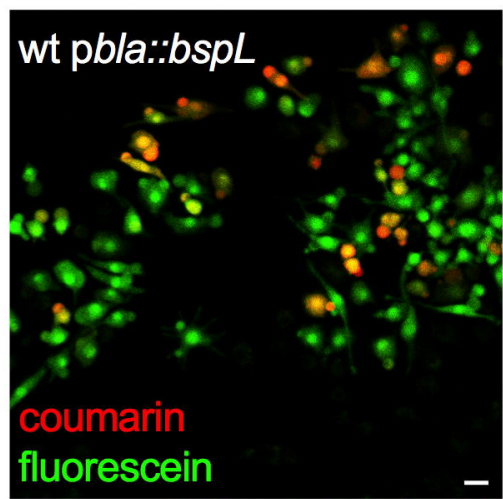

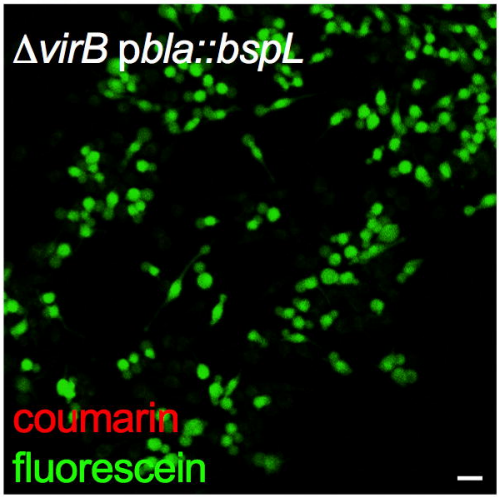


A

aCC-BY-NC-ND 4.0 International license.

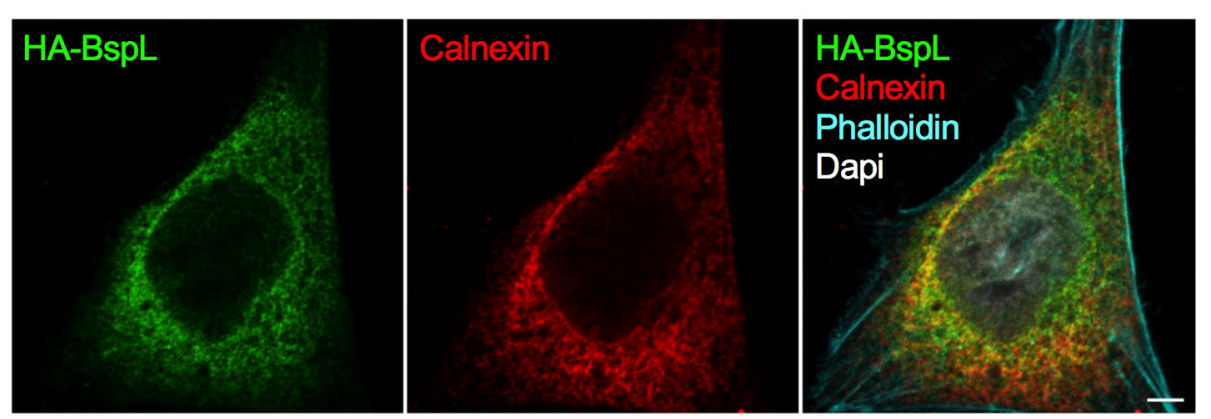

B

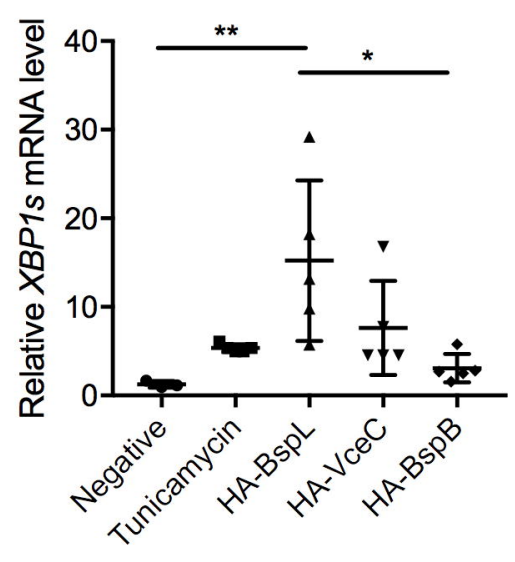

C

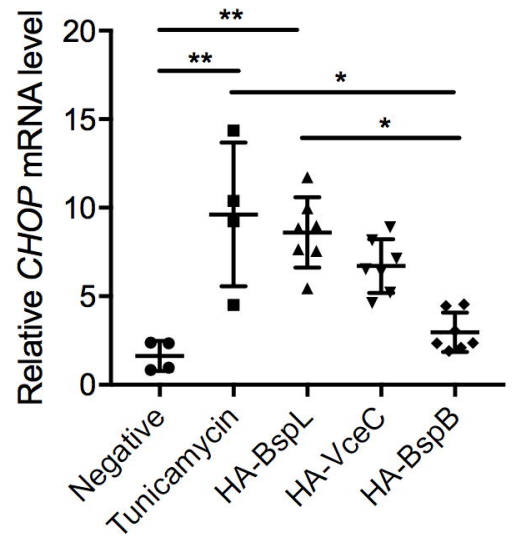

D

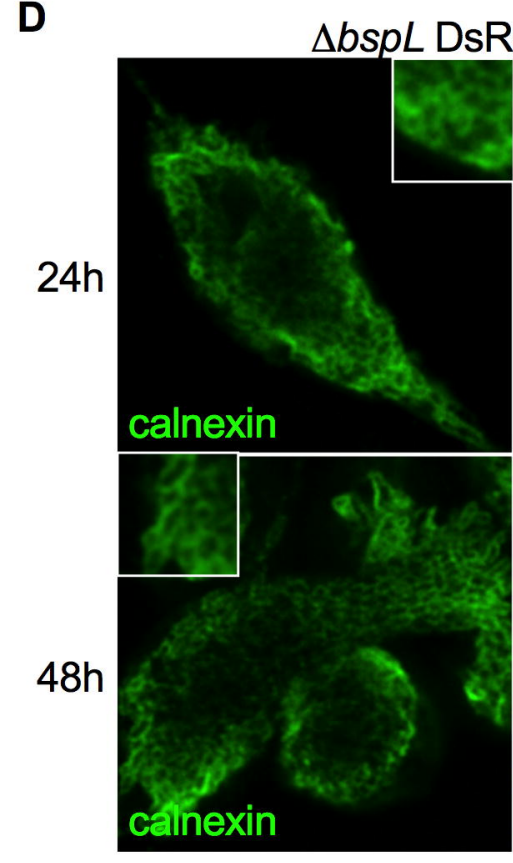

F

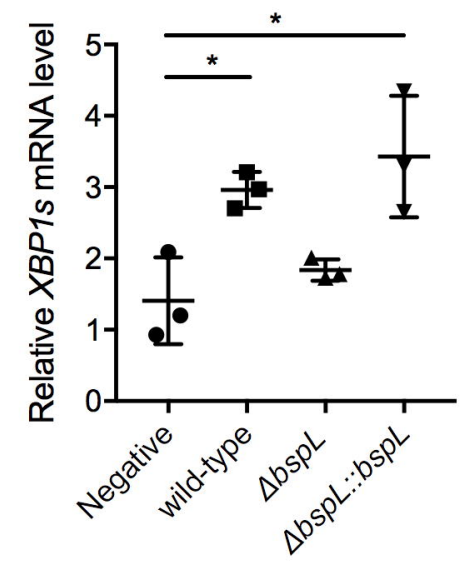

Figure 2
E

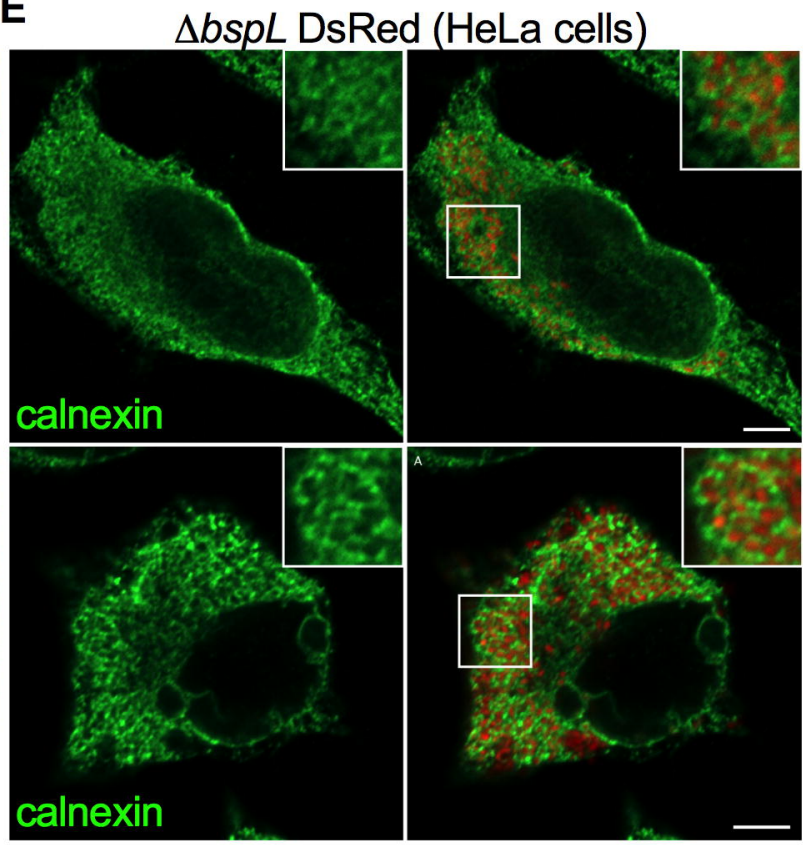

\section{G}

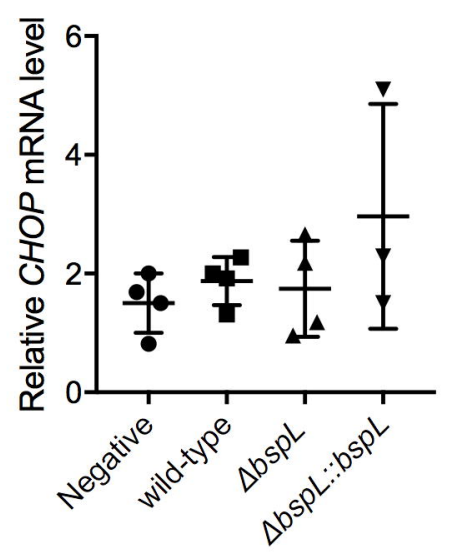




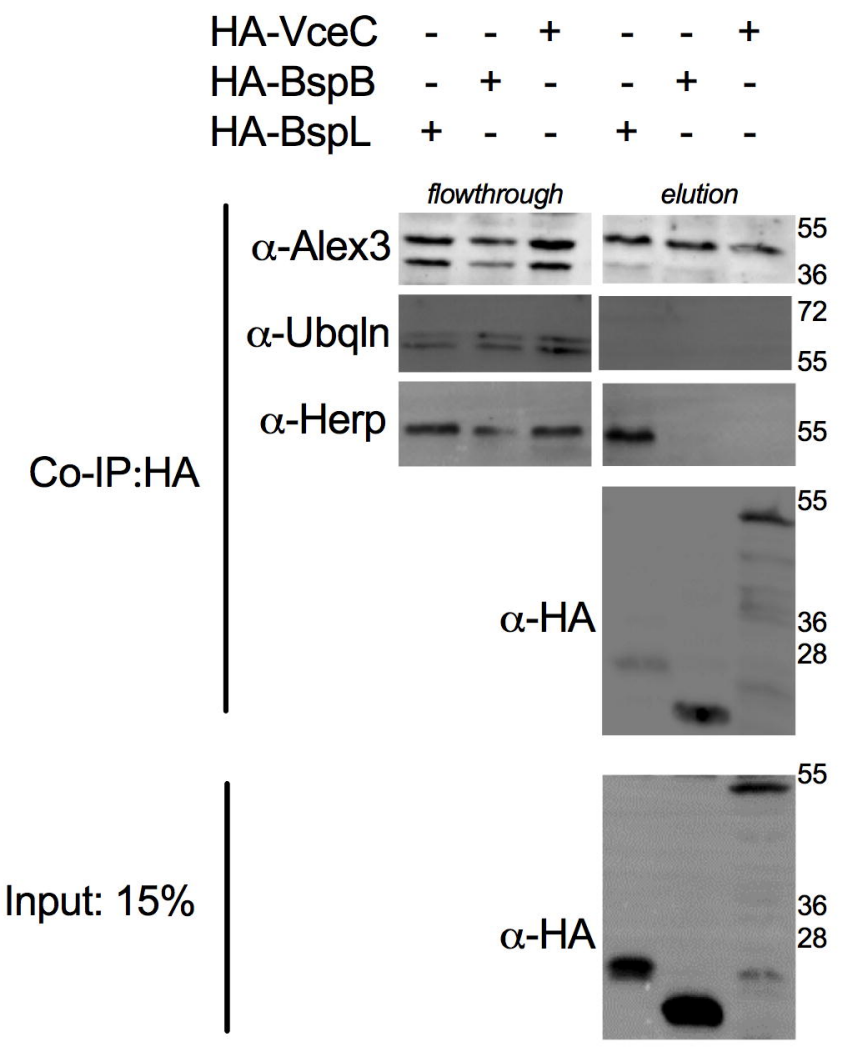

B

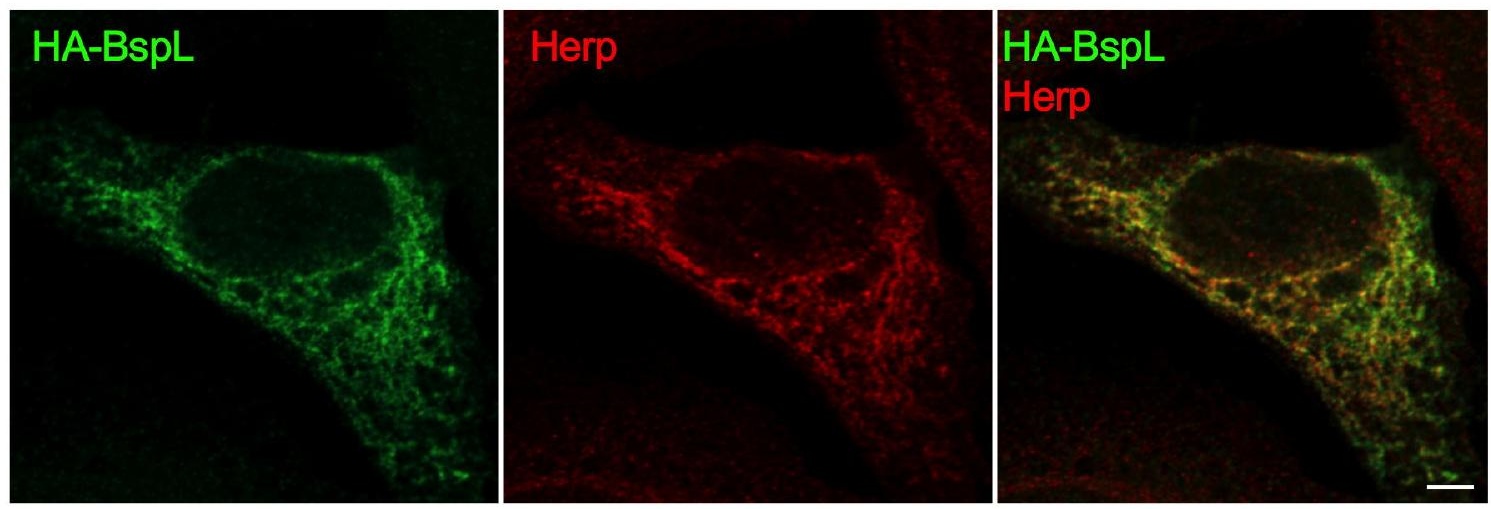

Figure 3 
A

B

HA-TCR $\alpha$ - $\quad+\quad+\quad+\quad+$

myc-Bspl - $\quad-\quad+\quad+$

cycloheximide $\quad-\quad-\quad+\quad-\quad+$

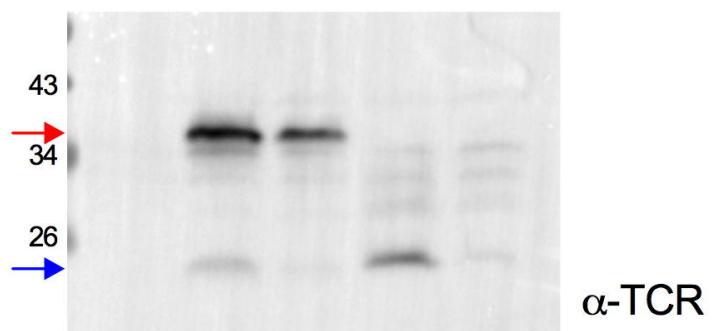

17

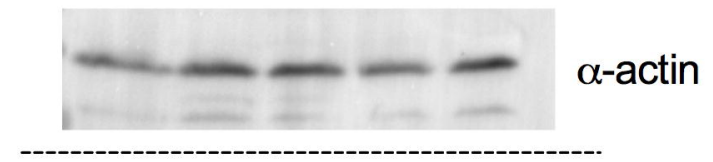

$\alpha-m y c$

$\alpha$-actin
$\mathrm{HA}-\mathrm{TCR} \alpha++\quad+$ myc-Bspl - $\quad+\quad+$ EndoH - + - +

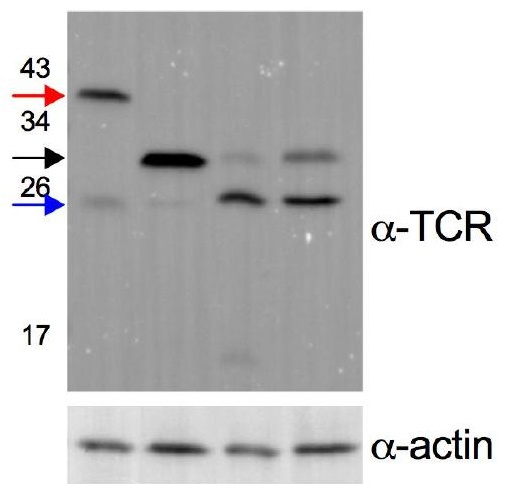

$\alpha$-actin

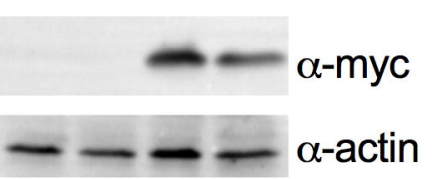

Figure 4 
A

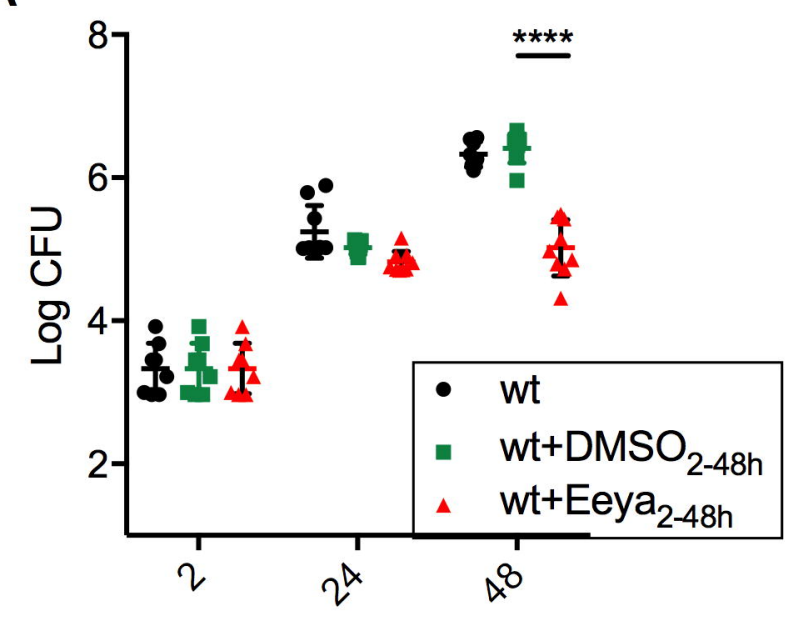

Time after infection (h)

B

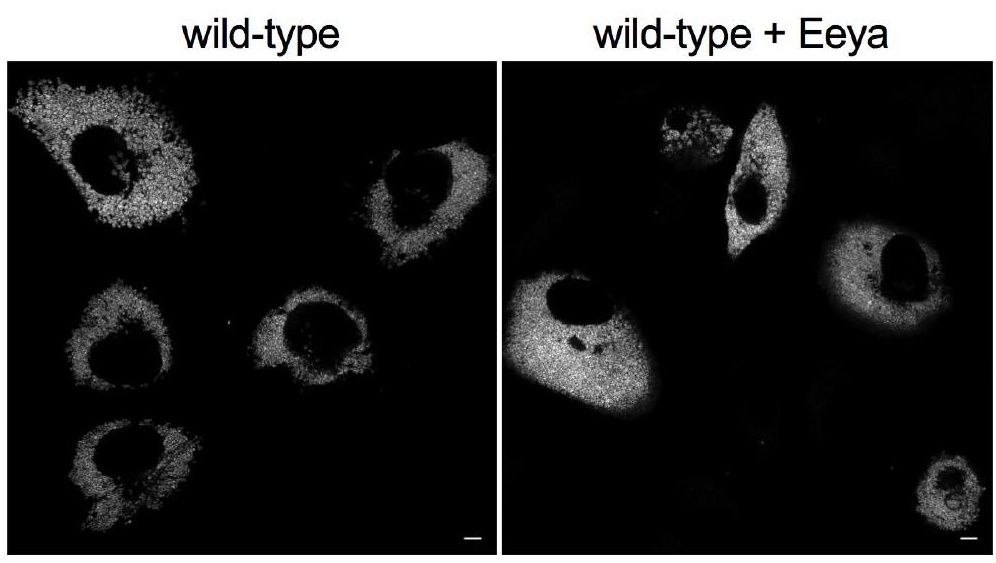

C

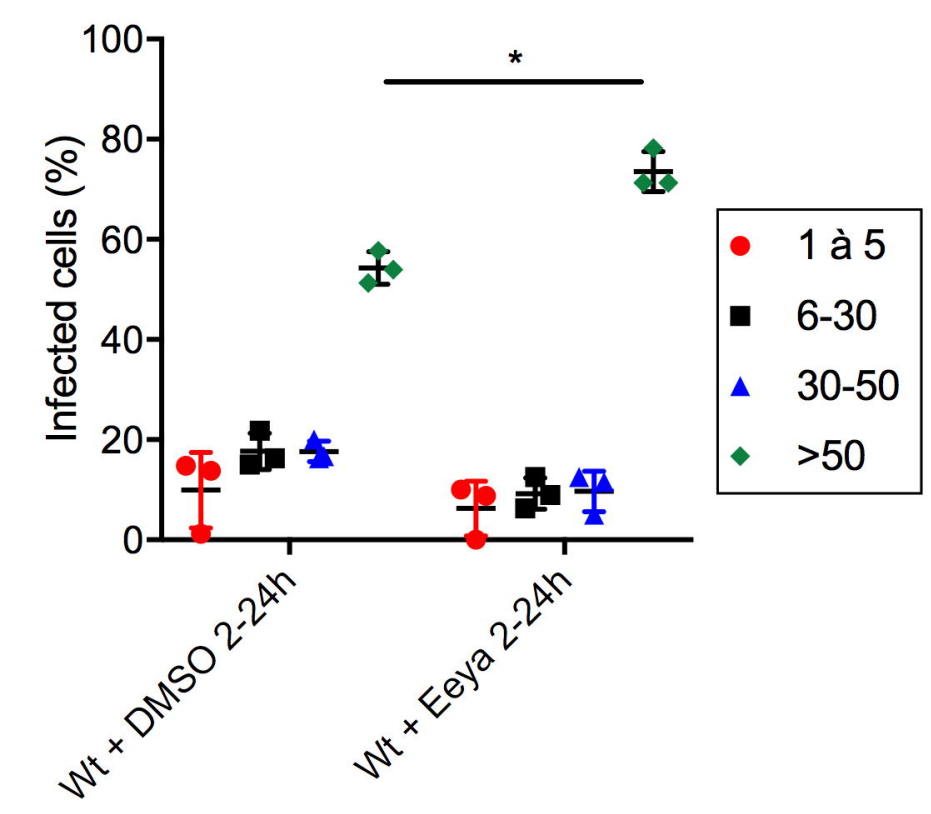

Figure 5 
A wild-type DsRed (iBMDM)

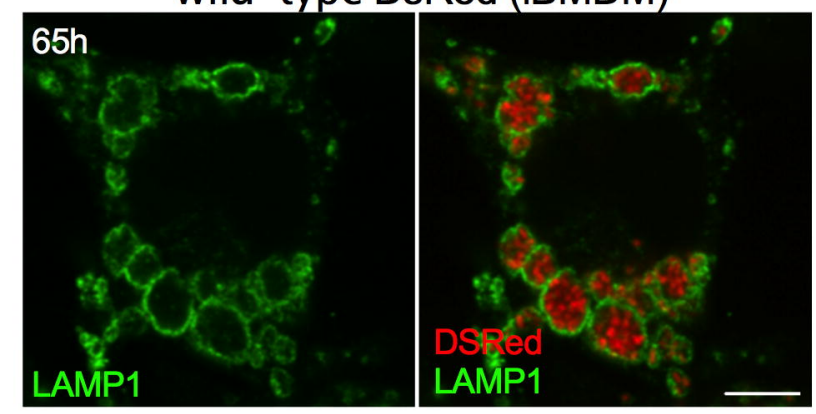

C

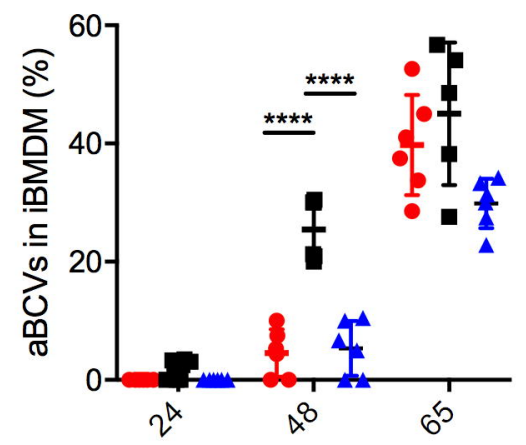

Time after infection (h)

\begin{tabular}{|ll|}
\hline - & wt \\
- & $\Delta b s p L$ \\
- & $\Delta b s p L:: b s p L$ \\
\hline
\end{tabular}

B

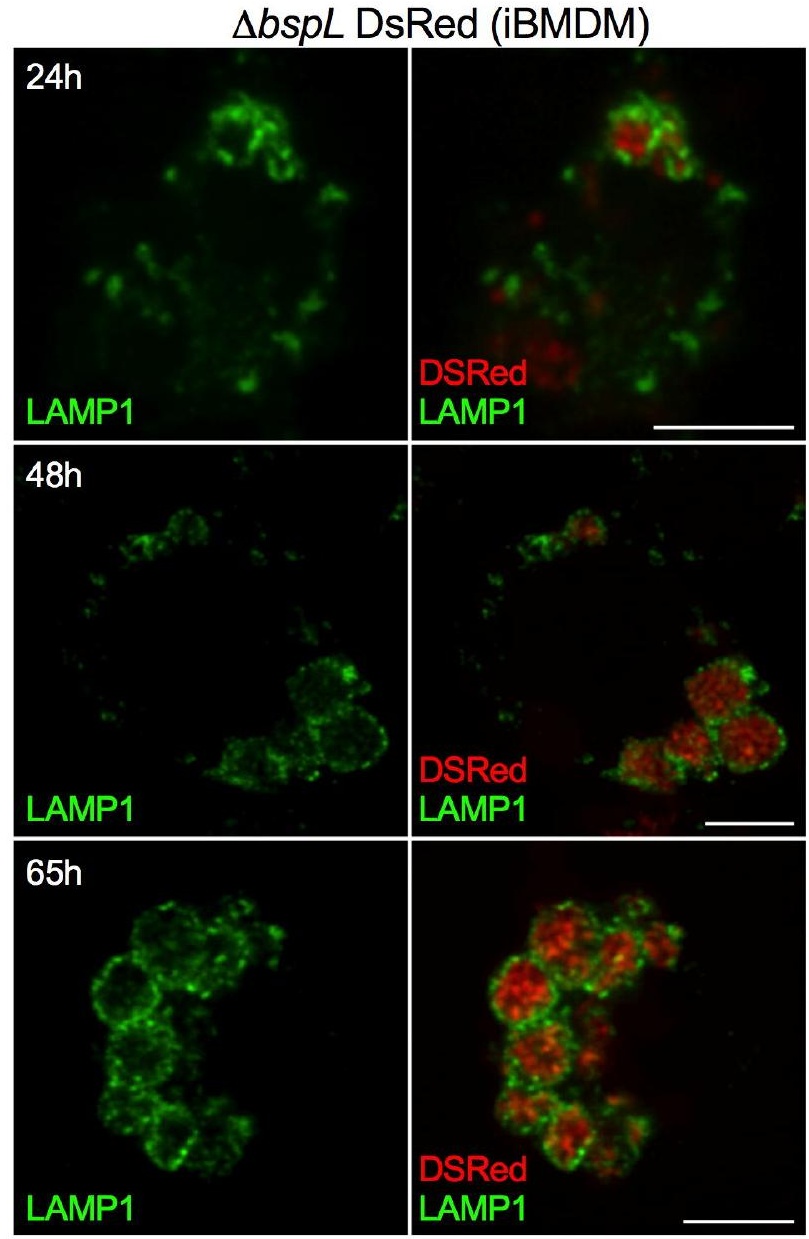

D

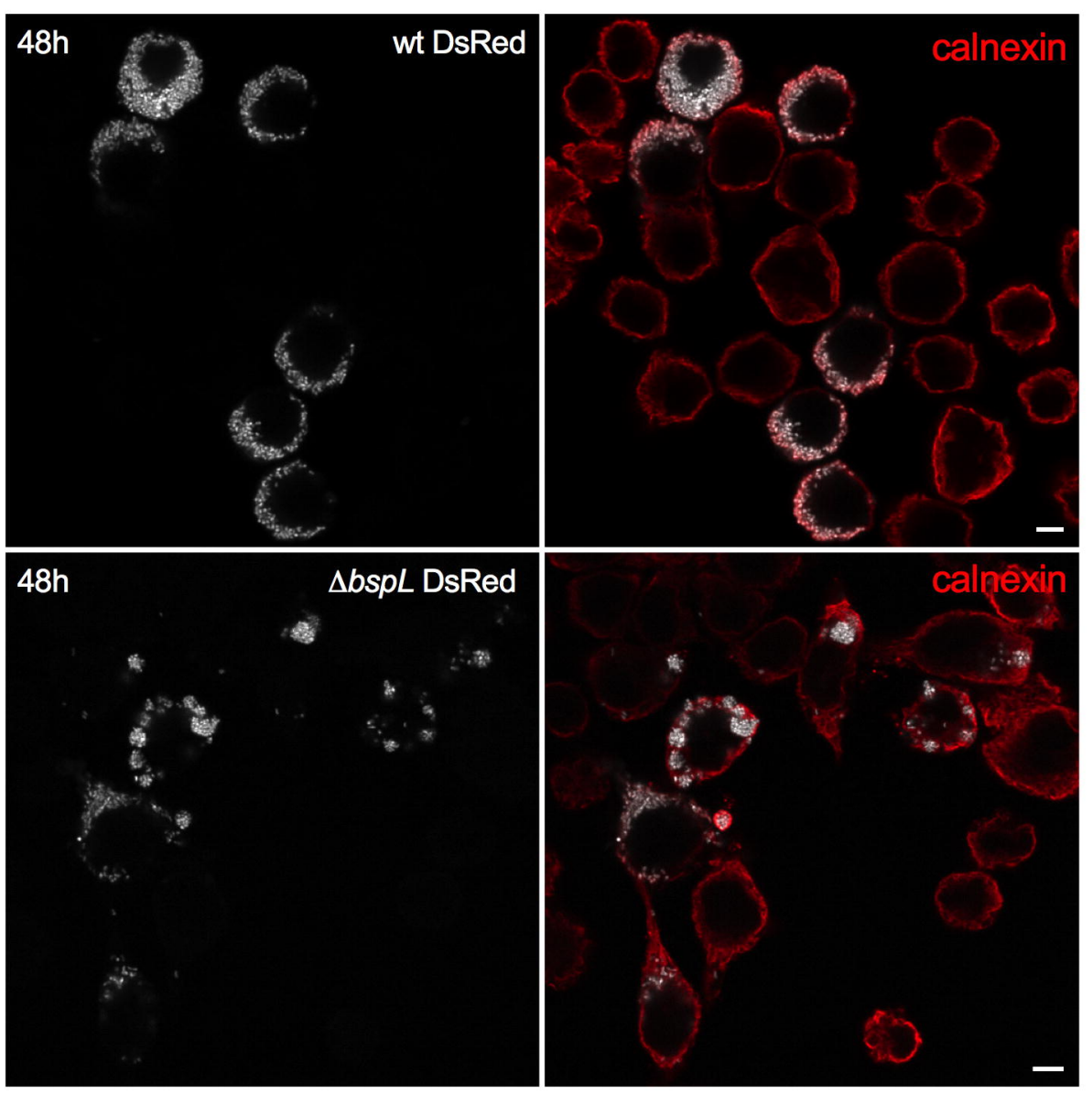

Figure 6 